Petkau et al., 04062021

\title{
The timing of differentiation and potency of CD8 effector function is set by RNA binding proteins
}

\author{
Georg Petkau', Twm J. Mitchell ${ }^{1}$, Krishnendu Chakraborty ${ }^{1}$, Sarah E. Bell ${ }^{1}$, Vanessa D'Angeli ${ }^{1}$, \\ Louise Matheson ${ }^{1}$, David J. Turner ${ }^{1}$, Ozge Gizlenci ${ }^{1}$, Fiamma Salerno ${ }^{1}$, Peter D. Katsikis ${ }^{2}$ and \\ Martin Turner ${ }^{1}$ \\ ${ }^{1}$ Immunology Programme \\ The Babraham Institute, \\ Babraham Research Campus, \\ Cambridge, CB22 3AT, \\ United Kingdom. \\ ${ }^{2}$ Department of Immunology, \\ Erasmus University Medical Center, \\ P.O. Box 2040, 3000CA Rotterdam, \\ Netherlands
}

Abstract

CD8 $\mathrm{T}$ cell differentiation into effector cells is initiated early after antigen encounter by signals from the $\mathrm{T}$ cell antigen receptor and costimulatory molecules. The molecular mechanisms that determine the timing and rate of differentiation however are not defined. Here we show that the RNA binding proteins (RBP) ZFP36 and ZFP36L1 limit the rate of differentiation of activated naïve CD8 T cells and the potency of the resulting cytotoxic lymphocytes. The RBP act in an early and short temporal window to enforce dependency on costimulation via CD28 for full T cell activation and effector differentiation by directly binding mRNA of NF-KB, IRF8 and NOTCH1 transcription factors and IL2. Their absence in T cells, or the adoptive transfer of a small numbers of CD8 T cells lacking the RBP, promotes resilience to influenza A virus infection without immunopathology. These findings highlight ZFP36 and ZFP36L1 as nodes for the integration of the early $T$ cell activation signals determining the speed and quality of the CD8 response.

Keywords: effector CD8 T cells, cytotoxicity, ZFP36, ZFP36L1, T cell activation, differentiation

\section{Introduction}

CD8 $T$ cells are instrumental for the clearance of pathogen infected or malignant cells and the provision of immune memory. Upon $\mathrm{T}$ cell receptor (TCR) sensing of peptide presented by MHC-I, naive CD8 $\mathrm{T}$ cells exit quiescence and engage a differentiation program to form cytotoxic T-lymphocytes (CTL) accompanied by massive clonal expansion. High affinity antigen overrides inhibitory mechanisms which ensure the quiescent state of the CD8 $\mathrm{T}$ cell and promotes growth and cell cycle entry $^{1,2}$. Co-stimulatory signals, termed "signal 2", from CD28 play an important role in lowering the activation thresholdespecially when TCR stimulation is suboptimal ${ }^{3}$.

The amount and duration of TCR stimulation correlates with the clonal expansion of antigen-specific cells ${ }^{4}$. However, weak TCR signals are sufficient to induce a full differentiation program, albeit more slowly ${ }^{5,6}$. The cytotoxic effector differentiation program is installed early after activation with estimates ranging between 2- and 48-hours after antigen stimulation ${ }^{4,7-10}$. Persistent exposure to IL-2, IL-12 and type-I interferon further shapes the response of CD8 T cells by providing critical survival 
factors and promoting effector differentiation ${ }^{11-13}$. The convergence of these signals on the regulation of transcription and chromatin states has been clearly demonstrated ${ }^{14-16}$. By contrast, an understanding of the nature and influence of RNA binding proteins (RBP) on CD8 $\mathrm{T}$ cell activation and differentiation is very limited ${ }^{17}$.

Amongst RBP, the ability of Roquin (Rc3h1) and Regnase1 (Rc3h12a) to limit CD8 T cell responses has been linked to the repression of TCR signaling and cytokine production ${ }^{18,19}$. Their absence in $T$ cells leads to $T$ cell hyperactivation and autoimmune/inflammatory disease. The ZFP36 family of RBP bind AU-rich elements (AREs) present in the 3' untranslated region (3'UTR) of mRNAs and can effect different outcomes promoting RNA decay ${ }^{19}$, suppressing translation ${ }^{17,20,21}$ or directing localised translation ${ }^{22}$ which are cell-context-specific ${ }^{23}$. Of the three ZFP36 gene-family members expressed by CD8 T cells, ZFP36L2 is present in naive and memory cells, while ZFP36 and ZFP36L1 are induced rapidly and transiently following TCR stimulation ${ }^{20}$. Zfp36-deficient mice show heightened immune responses and develop a severe autoimmune syndrome attributable to its role in myeloid cells ${ }^{19} 20$. An enhanced CD8 response in Zfp36-deficient mice has been linked to the excessive production by myeloid cells of IL-27, of which the p28 subunit is a direct target of ZFP36 ${ }^{24}$. In quiescent memory CD8 T cells the ZFP36-paralog ZFP36L2 suppresses the translation of cytokine $\mathrm{mRNA}^{25}$, but no studies have yet investigated the biology of ZFP36L1 in T cells. The widespread expression of ZFP36-family members by haematopoietic and mesenchymal cells and the genetic redundancy between them has made it challenging to understand the contributions of these genes to T cell physiology.

In this study we show ZFP36 and ZFP36L1 function during an early temporal window after activation in CD8 T cells to limit the tempo of effector differentiation and the functional capacity of differentiated effector cells. CTL deficient for both ZFP36 and ZFP36L1 show superior cell intrinsic cytotoxicity and confer greater protection against Influenza-A virus infection. A key function of ZFP36 and ZFP36L1 is to suppress T cell activation and enforce dependence upon costimulatory signals via CD28. The RBP bind transcripts encoding subunits of the NF-kB pathway, Notch1, IRF8 and II2 to regulate the abundance of their protein products early after $T$ cell activation. Thus, the acquisition of the CD8 effector fate is under the dominant control of ZFP36 and ZFP36L1.

\section{$\underline{\text { Results }}$}

ZFP36 and ZFP36L1 limit the anti-viral CD8 T cell effector response

To examine the consequences of the absence of ZFP36 and ZFP36L1 in T cells on an in vivo response we infected control mice $Z f p 36^{f / 1 / 1} / Z f p 36 / 1^{f / / 1 / 1}(\mathrm{WT}), \quad Z f p 36^{f / / 1 / 1} \mathrm{CD} 4^{\text {cre }}$ (ZFP36 KO),

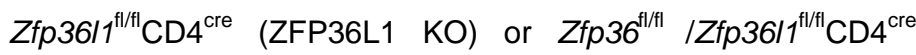
(dKO) mice intranasally with a sublethal dose of H1N1 Influenza A virus A/Puerto Rico/8/1934 (IAV/PR8). Following infection, using bodyweight as a surrogate for morbidity, we observe no significant difference between WT mice and mice with $T$ cells deficient for ZFP36 or ZFP36L1 (Fig.1a, b). By contrast, dKO mice show significantly less weight loss when compared to WT mice (Fig.1c). Moreover, when challenged with a lethal dose of IAV/PR8 dKO mice show increased survival compared to WT mice (Fig.1d). We conclude that the absence of ZFP36 and ZFP36L1 in T cells does not promote immunopathology, but rather increases resilience following infection with a pathogenic virus.

The presence of viral RNA in lung tissues of dKO mice following IAV/PR8 infection is reduced throughout the infection and by day 10 we found no detectable viral RNA in five of the ten infected dKO mice (Fig.1e). This is indicative of more efficient viral clearance when ZFP36 and ZFP36L1 are absent from T cells and may explain why the lungs of dKO mice contain reduced numbers of CD8 T cells specific for IAV nucleoprotein peptide $\mathrm{NP}_{(366-374)}$ compared to WT mice (Fig.1f). There was an increased frequency of granzyme-B (GzmB) positive antigenspecific CD8 $T$ cells in the lungs of dKO mice compared to WT mice at 4- and 10-days post-infection (Fig.1g). In addition, as shown by the increased GzmB staining intensity, dKO CD8 T cells contain greater amounts of GzmB per cell (Fig.1h) suggesting the potential for enhanced cytotoxic effector function. To determine the in vivo cytotoxicity of $\mathrm{CD}^{+} \mathrm{T}$ cells we cotransferred into IAV/PR8 infected or non-infected mice a 1:1 mixture of C57BL/6 splenocytes labelled with a low amount of 
bioRxiv preprint doi: https://doi.org/10.1101/2021.06.03.446738; this version posted June 4, 2021. The copyright holder for this preprint (which was not certified by peer review) is the author/funder, who has granted bioRxiv a license to display the preprint in perpetuity. It is made available under aCC-BY 4.0 International license.

Petkau et al., 04062021
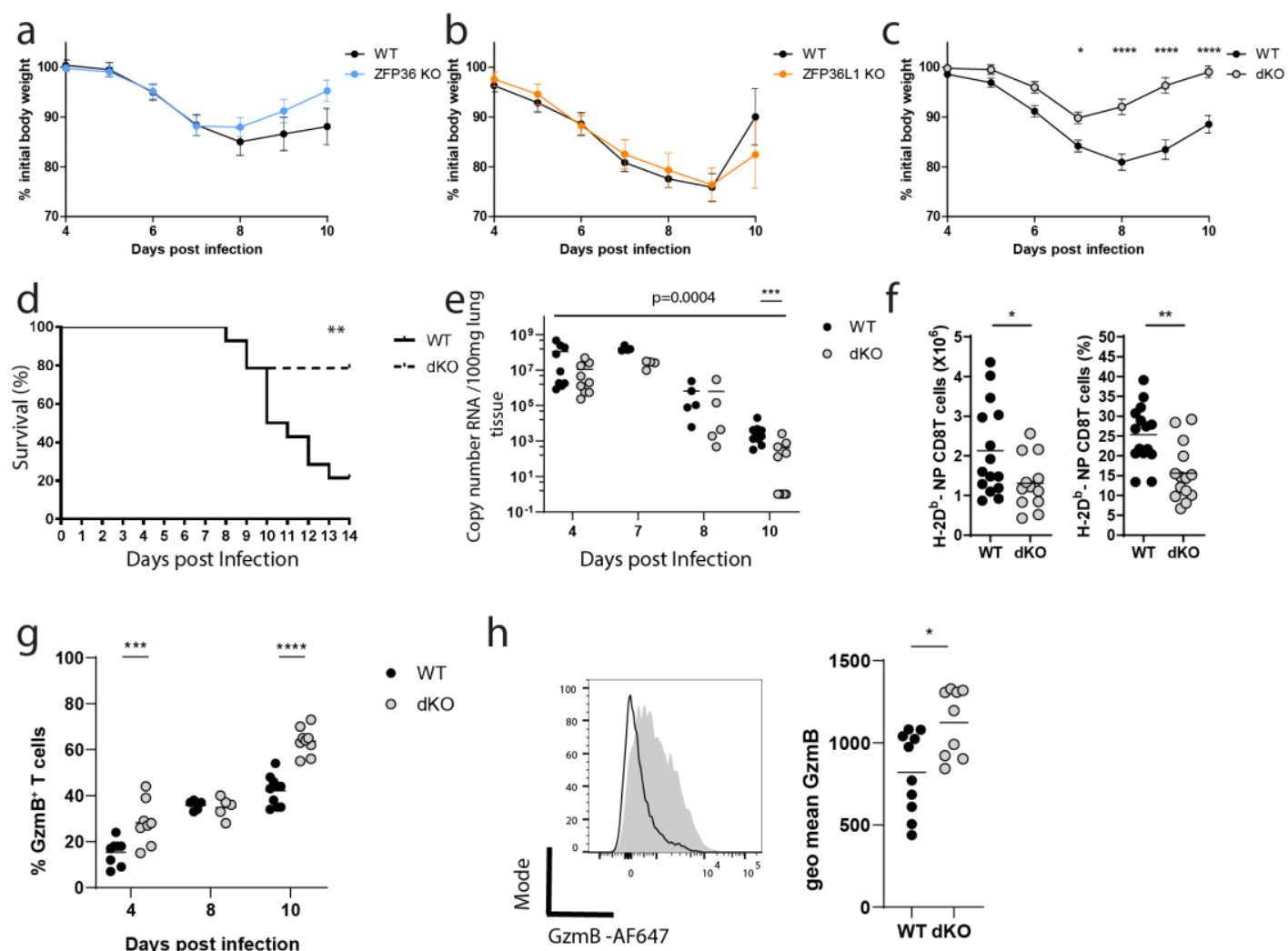

$\mathrm{h}$
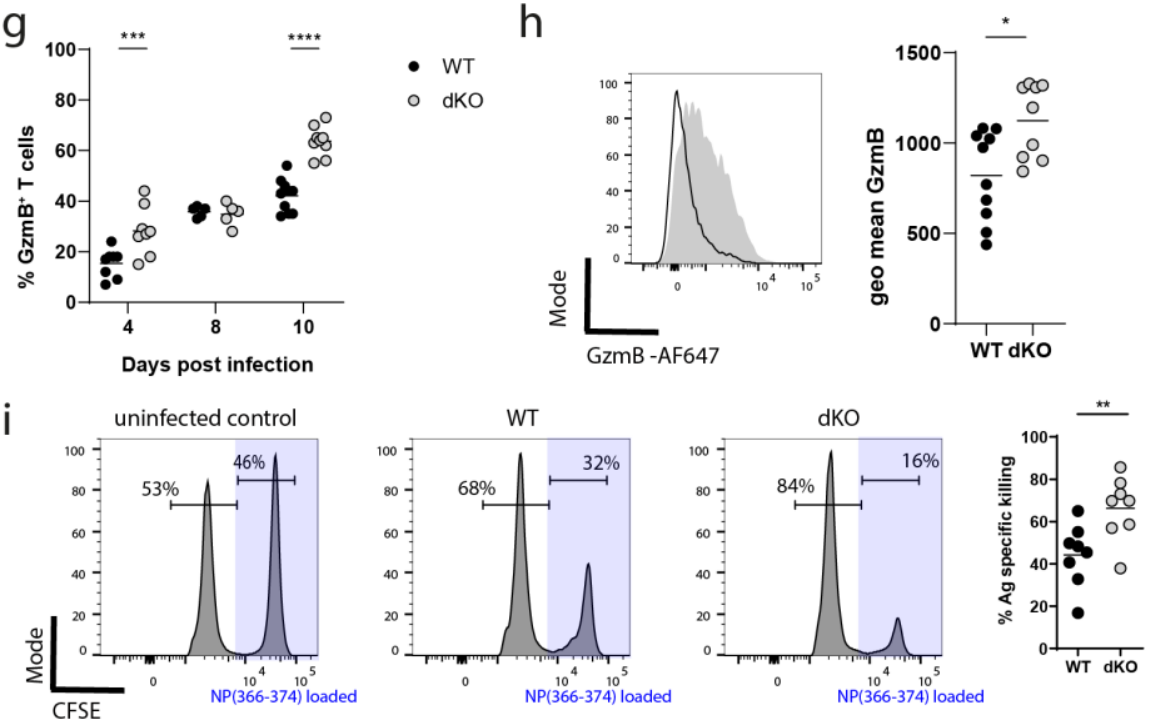

Fig. 1: ZFP36 and ZFP36L1 limit the anti-viral CD8 T cell effector response. Bodyweight loss as a percentage of initial body weight (100\%) in a) ZFP36 KO; b) ZFP36L1 KO; or c) dKO mice compared with age matched WT mice. $\mathbf{a}$ and $\mathbf{b}$ show combined data from of two independent experiments with $n=9-11$ mice per genotype for $c$ data is combined from four experiments with a total $n=20$ per genotype. Statistical significance determined by two-way ANOVA followed by Sidak's multiple comparisons test: ${ }^{*}=p<0.05 ;{ }^{* * * *}=p<0.0001$. Error bars indicate SEM. d) Survival curve of WT and dKO mice infected with a lethal dose of Influenza A virus; $n=14$ per genotype combined from two independent experiments. Statistical significance was tested by Mantel Cox rank test; ${ }^{*}=p<0.01$ e) Viral load in the lungs of mice at different time points following sub-lethal IAV infection. $n$ = 9 per genotype for day 4 and $7, n=5$ for day 8, and $n=10$ per genotype for day 10; Statistical significance was determined using a 2-Way ANOVA on log transformed data followed by Sidak's correction for multiple testing $<* * *=p<0.001$. pValue in panel shows the main Genotype effect. f) Relative and absolute numbers of virus specific CD8 T cells in lungs of mice on day 10 post infection. Statistical significance determined by an unpaired Student's ttest; ${ }^{*}=p<0.05$. Data is compiled from three independent experiments. $g$ ) Frequency of GzmB positive CD8 T cells recovered from lungs on day 4,8 and 10 after infection. Statistical significance was determined using a mixed-effects analysis followed by Bonferroni correction for multiple testing< ${ }^{* * *}=p<0.001,{ }^{* * * *}=p<0.0001$. h) Left) Representative overlay flow cytometry plot of GzmB staining on day 8 post infection. Open histogram represents WT and filled histogram dKO cells. Right) summary data for GzmB staining. Statistical significance determined by an unpaired Student's t-test; ${ }^{*}=\mathrm{p}<0.05$. i) In vivo cytotoxicity assay Left). Representative histograms showing proportions of labelled cells following transfer into uninfected C57BL/6 mouse (PBS) or day 10 IAV infected mice of the indicated genotype. Statistical significance determined by an unpaired Student's $\mathrm{t}$-test; ${ }^{* *}=\mathrm{p}<0.01$. In $\mathbf{e}$, $\mathbf{f}, \mathbf{g}, \mathbf{h}$ and $\mathbf{i}$ closed circles are the WT group and open circles dKO group with each symbol representing data from an individual mouse, of N>2 independent experiments. 
Carboxyfluorescein-succinimidyl-ester (CFSE) and CFSE-high splenocytes loaded with IAV $\mathrm{NP}_{(366-374)}$ peptide. The specific killing of peptide-loaded cells is dependent upon IAV/PR8 infection and is greater in dKO mice compared to WT mice

(Fig.1i). Taken together, these data show that the absence of ZFP36 and ZFP36L1 in T cells augments the cytotoxic effector function of MHC-I restricted CTL in vivo.

\section{Suppression of effector cell differentiation and function} mediated by ZFP36 and ZFP36L1 is cell intrinsic

To examine the intrinsic properties of naïve dKO CD8 T cells following adoptive transfer we generated mice with the OT-I TCR transgene which recognises the OVA $257-264$ peptide (SIINFEKL) presented by $\mathrm{H}-2 \mathrm{~K}^{\mathrm{b}}$. Following transfer of naïve OT-I cells into CD45.1 $1^{+}$B6.SJL (Ptprc ${ }^{\mathrm{a}} \mathrm{Pepc}^{\mathrm{b} / \text { Boy }}$ ) mice and infection with influenza $A$ virus/WSN/33 expressing the SIINFEKL peptide (IAV/WSN-OVA) the descendants of transferred cells are identified as CD45.2 $2^{+}$. Strikingly, in mice that had received 200 naïve OT-I cells lacking both Zfp36 and Zfp36/1 the body weight loss that accompanies IAV-infection is significantly reduced compared to mice which received the same number of WT cells

(Fig. 2a). Consistent with our studies in intact mice there are fewer dKO OT-I cells in the lung and the spleen at day 10 of infection than in mice with transferred WT OT-I cells (Supplementary figure 1a, b). In the lungs the progeny of transferred dKO cells are skewed towards a KLRG1+ ${ }^{+}$IL7R short lived effector (SLEC) phenotype compared to WT cells 10 days after infection (Fig.2b). In line with this, the dKO OT-I cells in the lung (Fig.2C) and spleen (Fig.2d) of infected mice at day 10 contained higher frequencies of TNF- and IFN $\gamma$-producing cells than WT OT-I cells. Moreover, frequencies of Granzyme-B positive dKO cells are increased in lung (Fig.2e) and spleen (Fig.2f) of infected mice compared to WT. This indicates the enhanced effector differentiation of naïve CD8 T cells lacking Zfp36 and Zfp36/1 is independent of their roles in other cells.

We used an adoptive transfer model with attenuated Listeria monocytogenes (attLm) expressing OVA to monitor in the same mouse the kinetics of differentiation of naïve OT-I cells. On the day following transfer of naive OT-I cells into B6.SJL mice we infected them by the intravenous route and sampled cells in the blood thereafter. The dKO OT-I cells accumulate slightly fewer cells than WT OT-I (Supplementary figure 1c) with the population skewed towards SLEC (Supplementary figure 1d). After peptide re-stimulation ex vivo, dKO OT-I cells show an increased proportion of TNF- and IFN $\gamma$-producing cells compared to WT OT-I cells (Supplementary figure 1e). Furthermore, dKO OT-I cells produced greater amounts of TNF per cell than the WT OT-I (Supplementary figure 1f). Thus, in model viral and bacterial infections, Zfp36 and Zfp36/1 act within CD8 T cells to delay the onset and limit the magnitude of acquired effector functions. 


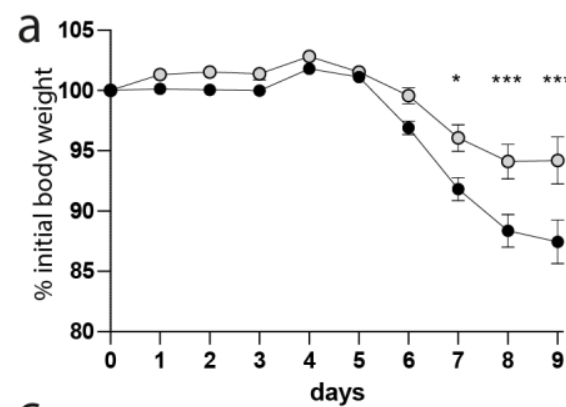

C
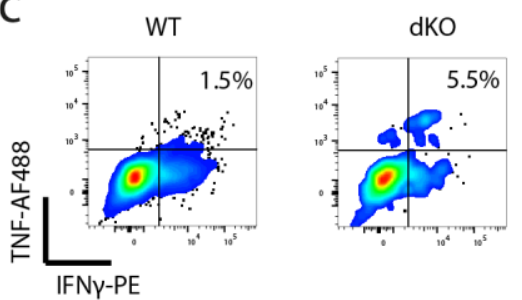

e

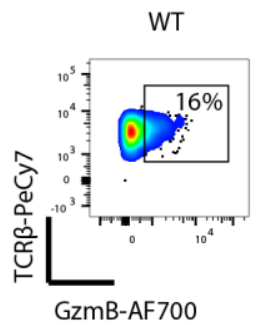

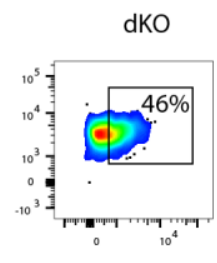

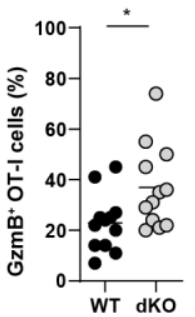

b
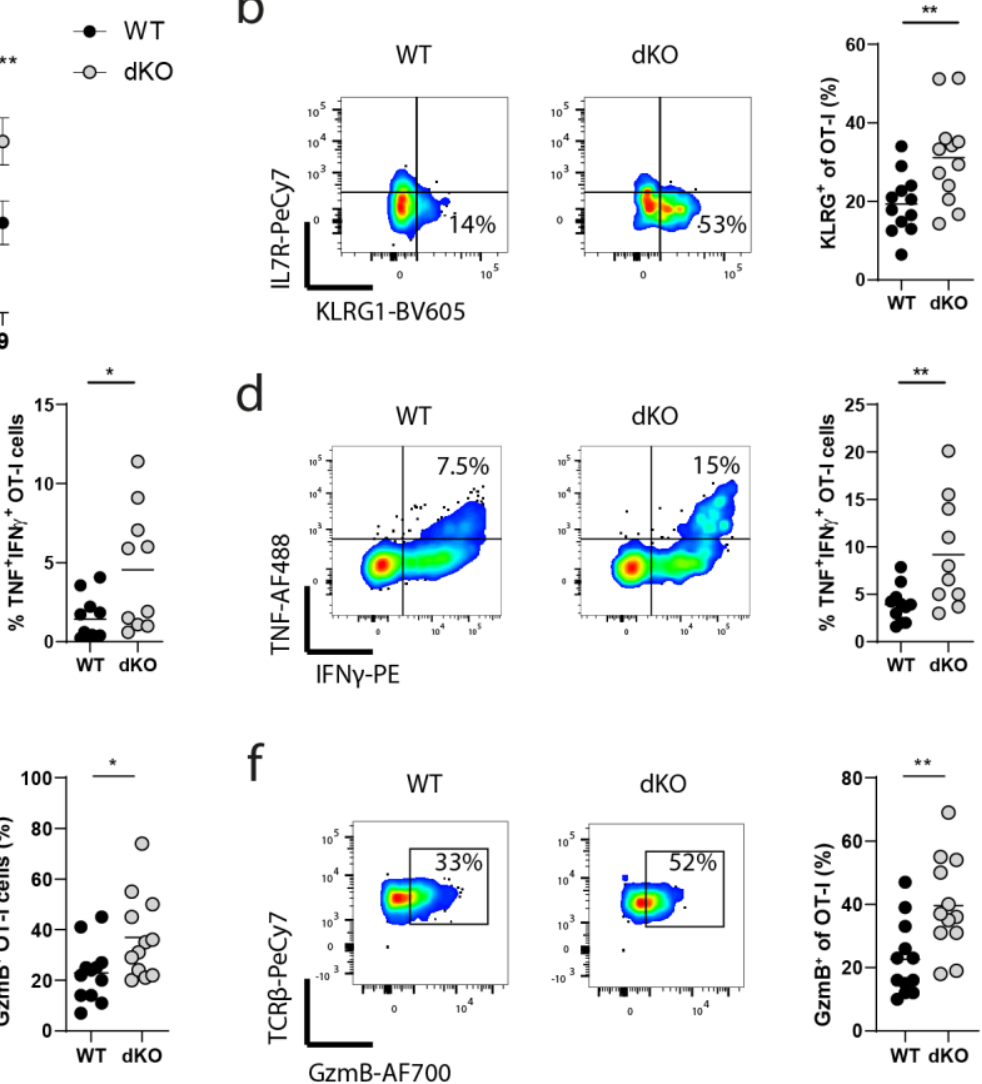

Fig. 2: Suppression of effector cell differentiation and function mediated by ZFP36 and ZFP36L1 is cell intrinsic. a) Body weight loss of WT mice which received 200 WT or dKO naïve OT-I cells the day before infection with a sublethal dose of WSN-OVA virus. Data is compiled from four independent experiments with the following number of mice per day: day 0-3 WT $n=12$, dKO $n=11$; day 4-8 WT $n=29$, dKO $n=30$; day 9 WT $n=20$, dKO $\mathrm{n}=23$; Statistical significance was tested using two-way ANOVA followed by Sidak's post-test for multiple comparisons; ${ }^{*}=\mathrm{p}<0.05,{ }^{* *}=\mathrm{p}<0.01$, ${ }^{* * *}=p<0.001$; error bars indicate SEM. b) Representative flow cytometry (left panel) and frequencies (right panel) showing relative abundance of SLEC among transferred OT-I cells recovered from lungs on day 10 post infection. Data is compiled from two independent experiments. Representative flow cytometry and frequencies of TNF and IFN $\gamma$ producing OT-I cells in c) lung and d) spleen 10 days after infection. Representative flow cytometry plot and frequencies of GzmB positive cells among OT-I cells in the lung f) and spleens g) 10 days after infection. Data is compiled from two independent experiments. Statistical significance was tested using an unpaired Student's t-test; ${ }^{*}=p<0.05,{ }^{* *}=p<0.01,{ }^{* * *}=p<0.001$. In all panels closed circles are the WT group and open circles the dKO group with each symbol representing data from an individual mouse. 


\section{ZFP36 and ZFP36L1 determine the CD8 effector program} early after $\mathrm{T}$ cell activation To discriminate the roles of the RBP during activation of naive CD8 T cells from their roles in limiting effector function in differentiated CTL, we activated OT-I cells in vitro with peptide in the presence of IL-2 and IL-12 for 24 hours and cultured them with IL-2 for seven days to generate CTL. We generated CTLs from OT-I-Zfp36 $6^{f / / 1 / 1} / Z f p 36 / 1^{f / / 1 / 1} \mathrm{CD} 4^{\text {cre }}$ mice where ZFP36 and ZFP36L1 are absent during initial T cell activation. We compared these with CTLs generated from OT-I-Rosa26Lstop_L-Cas9GFP-CD ${ }^{\text {cre }}$ mice transduced at 24 hours by viruses expressing sgRNAs targeting Zfp36 and Zfp36/1. In the latter, both RBPs are present in OT-I cells during initial activation but

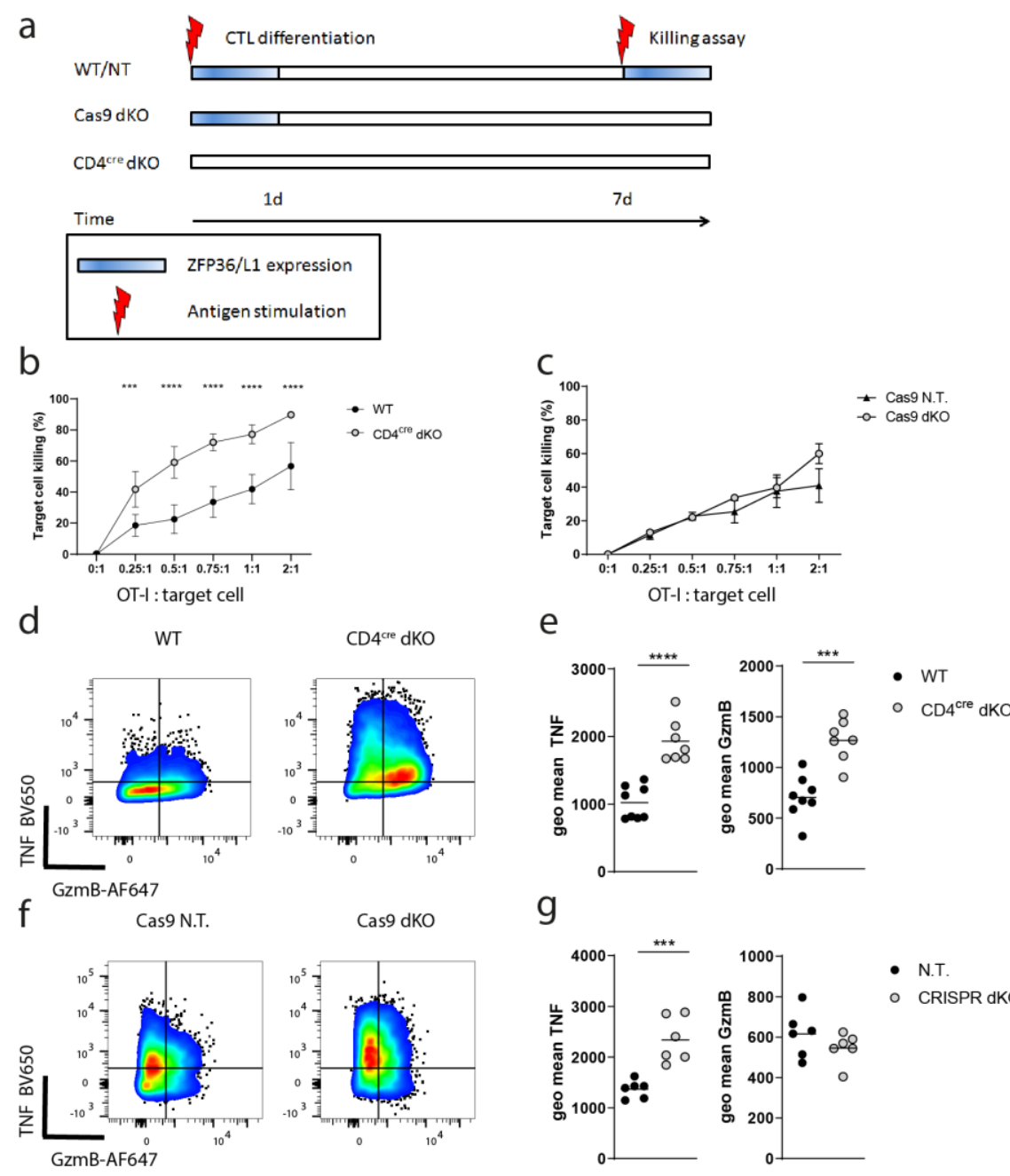

absent in CTLs (Fig.3a). Consistent with this expectation the efficiency of knockout of ZFP36 and ZFP36L1 with CRISPRCas9 was very high as evident from Western blotting of CTL whole cell lysates (Supplementary Figure 2a-d).
The CTL generated from OT-I-Zfp36 $6^{\mathrm{fl/fl}} / Z \mathrm{Zfp} 36 / 1^{\mathrm{fl/fl}} \mathrm{CD} 4^{\text {cre }}$ mice show increased cytotoxicity against SIINFEKL peptide-loaded EL4 cells compared to that of CTL derived from WT mice (Fig.3b). By contrast, the CTL generated by Cas9-mediated knockout of Zfp36 and Zfp36/1 were not more potent than CTL generated following transduction with viruses expressing nontargeting sgRNAs (Fig.3c). Upon $\mathrm{T}$ cell stimulation the expression of Granzyme-B and TNF is increased when Zfp36 and Zfp36/1 were absent before $T$ cell activation (Fig.3d, e). Notably, CTL produced increased TNF upon restimulation irrespective of the mode of genetic modification (Fig.3f, g) indicating a role for the RBP in directly targeting and limiting TNF

Fig. 3: ZFP36 and ZFP36L1 determine the CD8 effector program early after $\mathbf{T}$ cell activation. a) Experimental design: In WT cells or cells transduced with non-targeting guides the ZFP36 and ZFP36L1 proteins are transiently expressed after $\mathrm{T}$ cell activation prior to transduction as well as upon restimulation of CTL. Cells which have undergone Cas9 mediated deletion of ZFP36 and ZFP36L1 lack both proteins upon restimulation but not during the initial activation period. $C D 4^{\text {cre }}$ mediated deletion generates naïve CD8 T cells which lack ZFP36 and ZFP36L1 during initial activation and re-stimulation. b) In vitro killing of N4 peptide loaded EL4 cells after three hours at indicated CTL to EL4 ratios, using CTLs with CD4 ${ }^{\text {cre }}$ mediated deletion of ZFP36 and ZFP36L1 and WT CTLs. c) Killing assay using CTLs which were transduced with non-targeting guides or guides targeting both ZFP36 and ZFP36L1 is shown. For $\mathbf{b}$ and $\mathbf{c}$ error bars indicate SD. Statistical significance was determined by one-way ANOVA followed by Tukey's test for multiple comparisons.

d) Representative FACS plot and fluorescence intensity e) of GzmB and TNF $\square$ expression after three hours of WT and $C D 4^{\text {cre }}$ dKO CTL exposure to peptide loaded EL4 cells at a 1:1 ratio. f) Representative FACS plot and fluorescence intensity g) of GzmB and TNF $\square$ expression after three hours of CTL, treated with non-targeting and ZFP36 and L1 specific guide RNAs, exposure to peptide loaded EL4 cells at a 1:1 ratio. For $b-g$ the data is representative of $n=7 / 8$ biological replicates from five independent experiments for $\mathrm{CD} 4^{\text {cre }}$ and WT. For sgRNAs targeting ZFP36 and ZFP36L1 or non-targeting controls the data is representative of two independent experiments, each with three independent pairs of sgRNAs. Statistical significance was determined by a paired students t-test; ${ }^{*}=p<0.05 ;{ }^{* *}=p<0.01 ;{ }^{* * *}=p<0.001$. 
in differentiated CTL. By contrast, there was no difference in the amount of Granzyme-B in CTL when ZFP36 and L1 were absent as a consequence of Cas9 mediated deletion. These findings suggest that the increased cytotoxicity and Granzyme-B expression of CTL lacking Zfp36 and Zfp36/1 is not due to the absence of the RBP in CTL per se, it reflects a specific role for these RBP during early activation in naïve $T$ cells as repressors of the CTL differentiation program.

\section{Dynamic gene expression networks targeted by ZFP36 and ZFP36L1 early after CD8 T cell activation}

To identify the direct targets of the ZFP36-family which act early in CD8 T cells to regulate the CTL differentiation we employed an approach that was agnostic to whether this was mediated by effects on RNA stability, localisation or translation. We searched for transcripts strongly ( $\log 2 \mathrm{FC}>1.3$ or $<-1.3$; p. adj. Value $<0.05$ ) induced or repressed following CD8 T cell activation for six- and 18-hours ${ }^{26}$. To identify direct targets of the RBP we performed ZFP36L1 "individual-nucleotide resolution UV crosslinking and immunoprecipitation" (iCLIP) on OT-I CTLs stimulated with peptide for three hours and combined this with "high-throughput sequencing of RNA isolated by crosslinking immunoprecipitation" (HITS-CLIP) from CD4 $\mathrm{T}$ cells activated for 4 hours $^{20}$. This yielded a unified list of 1147 candidate genes which were present in either or both data sets. The intersection of the dynamically regulated transcripts in CD8 T cells with this list identified 204 candidates for direct regulation by ZFP36 and ZFP36L1. Targets induced at 6 and/or $18 \mathrm{~h}$ post activation comprised the majority with 166 genes (81\%) of which 67 transcripts are shared between both time points (Fig.4a).

The target mRNAs could be broadly clustered into five groups according to their expression dynamics following activation (Fig.4b; Supplementary Figure 3a, b; Supplementary Table 1). The group I cluster includes genes that are induced early and transiently following $\mathrm{T}$ cell activation and include transcription factors of the NF- $\mathrm{B}$ B pathway and genes involved in signalling and protein synthesis (Supplementary Figure 3c). This group includes genes involved in metabolism (Slc7a1/5) and Kdm6b encoding a Histone-lysine specific demethylase. Group II comprises genes induced by six-hours and maintained high expression at 18 hours; it contains cytokines and chemokines which include IL-2, Ifng, Tnf, Cc/3 and Ccl4. Genes involved in protein synthesis, signaling, transcription factors such as $c-M y c$, Zeb2, Irf4 and Irf8 with known roles in promoting CTL differentiation are also represented in this group. Prominent amongst genes induced 18 hours after activation in group III were ZFP36-family bound mRNAs encoding Cdk1, Cdk6 and Cdc45 which are associated with cell division. Cluster IV and V contain candidate target genes which are repressed by 6 hours or 18 hours post activation respectively relative to their expression in non-activated naïve cells. Some of these targets such as Tagln2 or Vim are functionally associated with signalling and cytoskeleton remodelling. The transcription factors Fos and Gata3 as well as Zfp36 are also among group V of target mRNAs being repressed at $6 \mathrm{~h}$ and 18 post activation (Supplementary Table 1). The latter two genes are well-known immediate early genes and present in the clusters of repressed genes as a consequence of their strong and transient transcriptional induction at timepoints earlier than six-hours. These data suggest that the RBPs can act consecutively on multiple functionally diverse target transcripts over the first 24 hours of activation. As the effector differentiation is accelerated in the absence of ZFP36 and ZFP36L1 we focussed on the direct regulation of genes linked to the acquisition of effector function. 
a

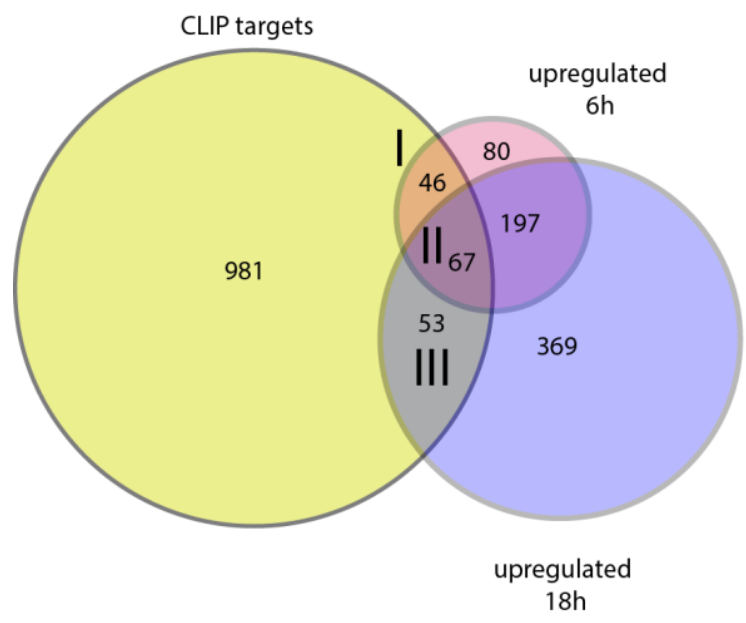

b

(I) Transient induced targets

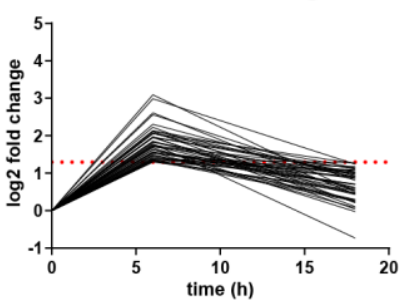

(III) Late induced targets

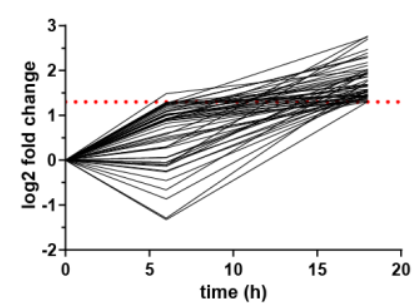

Fig. 4: Dynamic gene expression networks targeted by ZFP36 and ZFP36L1 early after CD8 T cell activation. a) Overlap of transcripts significantly differentially expressed (adjusted $p$ value $<=0.05$ ) by log2FC $>1.3$ or $<-1.3$, following activation of naive CD8 T cells for 6 or 18 hours with CLIP targets of ZFP36 and ZFP36L1. b) Clusters I-III showing ZFP36 family mRNA target dynamics during T-cell activation.

\section{ZFP36 and ZFP36L1 limit the magnitude of IL-2 production}

IL-2, which plays an important role in effector $T$ cell differentiation, was identified as a direct target of ZFP36 and ZFP36L1 in the CLIP analysis (Supplementary Fig.4a). However, the regulation of cytokines by ZFP36 has been shown to be cell context dependent ${ }^{23}$ and previous studies found that the absence of Zfp36 in CD8 T cells did not affect IL-2 production $^{24}$. Therefore we sought to examine IL-2 production by dKO cells using a sensitive bispecific antibody-based capture method ${ }^{27}$ which measured IL-2 secretion at the single cell level in the absence of a secretion inhibitor. Following peptide-stimulation the frequency of WT and dKO CD8 T cells producing IL-2 peaked around six-hours following peptide-mediated $\mathrm{T}$ cell activation.

(Fig.5 a, b). We found a substantial increase in the proportion dKO OT-I cells producing IL-2 compared to WT OT-I cells during the first 16 hours (Fig.5a, b). However, by 24 hours the frequency of IL-2 expressing cells is low and does not differ between $\mathrm{dKO}$ and WT cells. Therefore, the suppressive mechanisms that lead to the cessation of IL-2 production are intact in the absence of the RBP. In contrast we find the same staining intensity for CD25 between WT and dKO OT-I cells during the first $16 \mathrm{~h}$ after activation. However, expression of CD25 persists on dKO cells at $24 \mathrm{~h}$ a time at which it shows diminished expression on WT cells (Fig.5c).
For the WT cells the intensity of IL-2 staining showed little difference at any of the timepoints tested before 24 hours after activation. This is indicative of the "all-or-non" digital response to $T$ cell stimulation. By contrast, at three and six hours after activation, OT-I cells lacking ZFP36 and ZFP36L1 stain more intensely than WT OT-I for IL-2 indicating that they produce more IL-2 per cell (Fig.5d). Thus, early after activation ZFP36 and ZFP36L1 limit the frequency of activated T cells producing IL-2 and the amount of IL-2 produced by the activated CD8 T cell.

\section{ZFP36 and ZFP36L1 directly limit the expression of NF-KB, IRF8 and Notch1}

Multiple transcripts encoding components of the canonical and noncanonical NF-kB pathway, including Nfkb1, Nfkb2, Nfkbib and $R e l$ are induced at 6 hours post-activation and cluster in group I. The NF-kB pathway is critical to integrate TCR signaling with CD28 costimulation and inflammatory cues. In concert with the NFAT/AP1 complex, NF-kB promotes the induction of IL-2 by $T$ cells ${ }^{3}$. Analysis of ZFP36-family interactions with RNA confirmed Nfkb1 (Supplementary Figure 4b), Nfkb2 (Fig.6a) and Rel (Fig.6b) mRNA to be directly bound with accumulation of reads at AU-rich elements (AREs) in their 3'UTRs that are highly conserved between mammalian species (Fig.6c, $d$ : Supplementary Figure 4c). Consistent with this interaction 
a

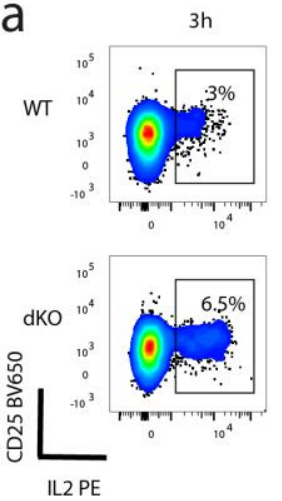

b

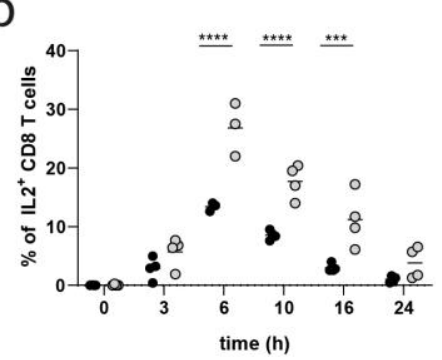

d

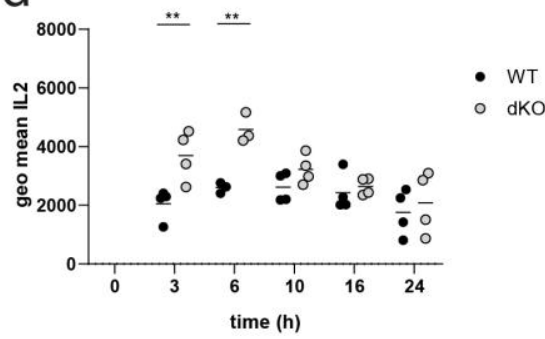

being consequential, we found that in CD8 T cells from dKO mice the abundance of representative members of the non-canonical

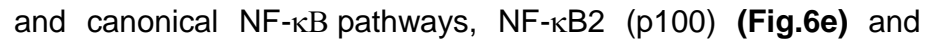
cREL (Fig.6f) is greater compared to WT following activation with anti-CD3. While NF-кB2 (p100) abundance remained greater in the dKO than the WT over the whole course of T cell activation, increased CREL protein was observed in the dKO only at early time-points.

The NF-kB pathway has been shown to interact with other transcription factors including $\mathrm{NOTCH} 1$ to drive differentiation into CTLs by directly regulating the expression of the transcription factor Eomesodermin (EOMES) ${ }^{28}$ and Granzyme$\mathrm{B}^{29}$. The Notch1 mRNA is bound by ZFP36 and ZFP36L1 in T cell CLIP data (Supplementary Figure 5a). This is consistent with an earlier biochemical demonstration of the interaction of ZFP36L1 with a 61 nucleotide sequence containing the Notch1
AREs $^{30}$. Although Notch1 is not among the mRNAs in the induced clusters, the expression of the $\mathrm{NOTCH} 1$ intracellular domain is increased in naïve dKO CD8 T cells at three-, six- and 18-hours post stimulation compared to WT cells (Supplementary Figure 5b, c).

TCR induced EOMES expression is NF-kB and $\mathrm{NOTCH}-1$ dependent and mediates the effector and memory differentiation program in CD8 $\mathrm{T}$ cells by promoting cytokine and granzyme expression ${ }^{28,29,31,32}$. We found dKO naïve CD8 T cells activated with plate bound anti-CD3 antibody showed greatly increased frequencies of Granzyme-B and EOMES positive cells compared to WT cells 72 hours after activation (Fig.6g, h). The increased expression of EOMES three days after activation was not due to relief of direct inhibitory effects of the ZFP36-family on the Eomes mRNA as it was not identified as a target mRNA. EOMES becomes highly expressed during the later course of the immune 


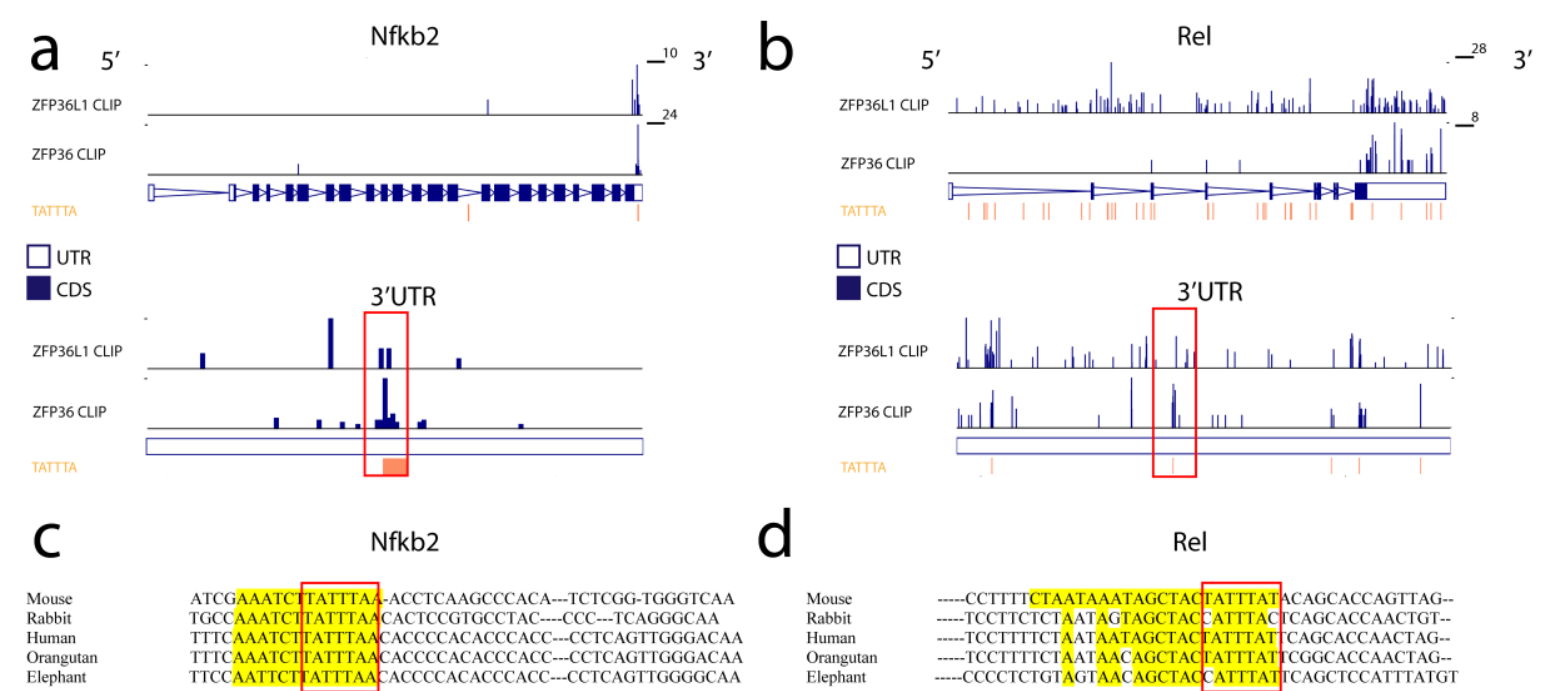

e

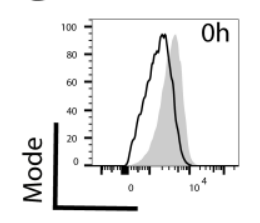

NFKB2-AF488

$f$
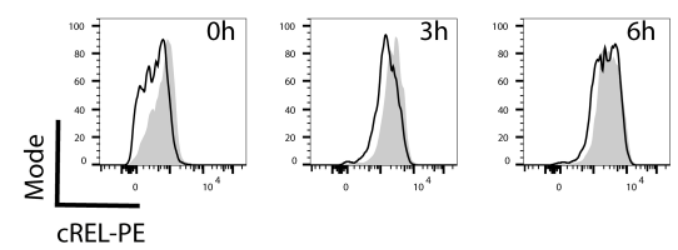

g

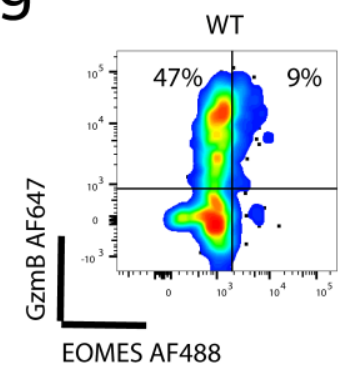

CREL

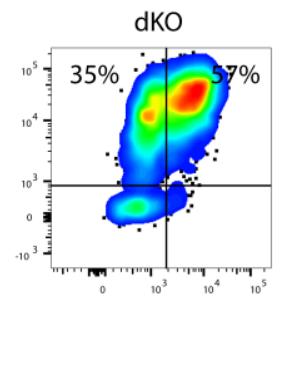

NFKB2 (p100)
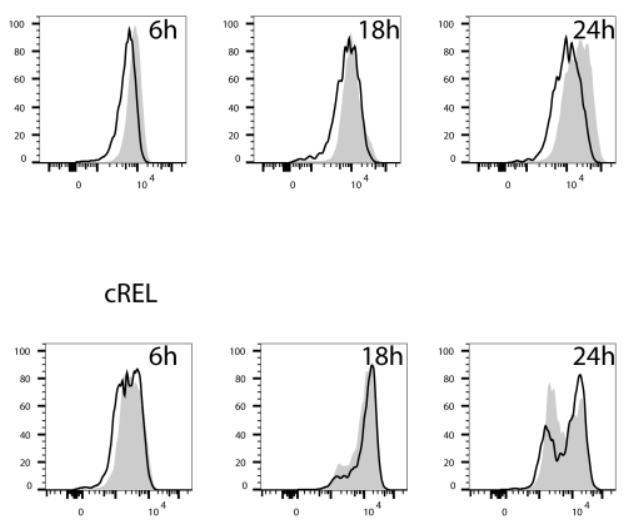

h

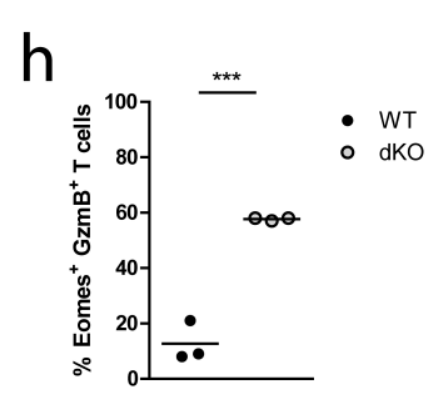

Fig. 6: ZFP36 and ZFP36L1 limit the early expression of transcription factors driving effector differentiation. a) CLIP data showing sequencing reads across a) Nfkb2 and b) Rel transcripts with an expanded view of the 3'UTR. Top lane shows ZFP36L1 CLIP data from OT-I CD8 CTLs stimulated for 3h with N4 peptide. Bottom lane shows ZFP36 CLIP data from in vitro activated naive CD4 T cells. TATTTA motifs are identified in orange; $\mathbf{c}$, $\mathbf{d}$ conservation among vertebrates of the ZFP36/ZFP36L1 binding site determined using MULTIZ alignment tool in UCSC genome browser for c) Nfkb2 and d) Rel. Binding as identified by CLIP is highlighted in yellow. Selected species are ordered from top to bottom according the evolutionary proximity of the clades. Red bracket highlights the ARE. For Rel the best conserved binding site is displayed. e) Differential protein expression of NF-kB2 and f) cREL by naive CD8 T cells following activation with plate bound anti-CD3 antibody. Representative flow cytometry histograms (left panel) and geo mean fluorescence (right panel) are shown. Open histograms represent WT and filled histograms show dKO cells. Statistical significance was determined using a mixed-effects model analysis followed by Sidak's correction for multiple testing; ${ }^{*}=p<0.05$. Data is compiled from two of three independent experiments. g) Expression of EOMES and GzmB by naive WT and dKO CD8 T cells activated with plate bound CD3 for 72h. h) Frequency of GzmB and EOMES expressing cells. Statistical significance was tested with unpaired Student's t-test; ${ }^{* * *}=p<0.001$. Data is compiled from two independent experiments and three biological replicates. 
response and not in naive $T$ cells $^{28}$. This data highlights the more rapid acquisition of an effector state in dKO cells mediated via targets accelerating EOMES expression. Group II transcription factors IRF4 and IRF8 have also been shown to be important for CTL differentiation and function ${ }^{33,34}$. In particular Irf8 has been suggested to act independently of T-box transcription factors Tbet and Eomes to promote effector functions ${ }^{33}$. Irf8 mRNA, which is rapidly induced upon $\mathrm{T}$ cell activation and continues to be highly expressed by $18 \mathrm{~h}$, is also bound by ZFP36 and ZFP36L1 in T cells (Supplementary Figure $\mathbf{5} \mathbf{d}$ ). IRF8 protein is increased in dKO cells compared to WT cells, particularly so at later time points of stimulation (Supplementary Figure 5 e, f). Taken together, these data suggest a collective of transcription factors known to form a network promoting $\mathrm{T}$ cell differentiation are regulated by ZFP36 and ZFP36L1.

\section{ZFP36 and ZFP36L1 inhibit TCR mediated T cell activation and promote dependence on CD28}

The dependency of T cells on CD28 is regulated by the NF- $\kappa B$ pathway $^{3}$ including NF-кB2 ${ }^{35}$. Also not only transcription of II2 is dependent on CD28 costimulation via $N F-\kappa B^{36}$ but $C D 28$ also promotes the stabilization of the $/ 12 \mathrm{mRNA}^{37}$. This prompted us to test the co-stimulation dependence of $\mathrm{dKO}$ CD8 $\mathrm{T}$ cells by the titration of anti-CD28 antibodies. Following stimulation with plate bound anti-CD3, cell trace labelled dKO CD8 T cells divide more than their WT counterparts and accumulate greater cell numbers $72 \mathrm{~h}$ after stimulation(Fig. 7a,b), demonstrating a reduced activation threshold in dKO cells. The addition of anti-CD28 (clone 37.51) increases the numbers of both, WT and dKO cells present after 72 hours culture, compared to cultures with just
anti-CD3 (Fig.7b). WT cells show a greater sensitivity to lower amounts of CD28 costimulation compared to the dKO CD8 T cells. The latter are only mildly responsive to the inclusion of antiCD28 as measured by the absolute cell numbers in the cultures after 72 hours with $\operatorname{LogEC}_{50}$ of $1.6 \mu \mathrm{g} / \mathrm{ml}$ for WT and $0.89 \mu \mathrm{g} / \mathrm{ml}$ dKO cells (Fig.7b). dKO cells accumulate more cells per generation, when stimulated with CD3 alone as compared to their WT counterparts (Fig.7c,d), which results in a reduced sensitivity to additional CD28 costimulation in dKO cells. This contrasts with WT cells which are critically dependent on signals from CD28, which opposes the inhibition of activation by ZFP36 and ZFP36L1, to enable a robust proliferative response.

As shown above stimulation of CD8 T cells with anti-CD3 alone induced significantly greater amounts of both NF-kB2(p100) (Fig.7 e, f) and IRF8 (Fig.7 g, h) in dKO cells compared to WT. The addition of $5 \mu \mathrm{g} / \mathrm{ml}$ plate bound anti-CD28 augmented the expression of both transcription factors, indicating they were responsive to co-stimulation and reduced the differences in NFкB2 and IRF8 expression between dKO cells and WT. Importantly, the increased expression of NF-KB2(p100) and IRF8 by CD8 T cells lacking ZFP36 and ZFP36L1 persisted upon addition of large amounts of recombinant IL-2 indicating these differences are not secondary to increased IL-2 production by the dKO T cells. Thus, ZFP36 and ZFP36L1 regulate CD28 costimulation also independently of the production of IL-2. The absence of ZFP36-family members in the naïve CD8+ $T$ cell reduces the requirement for CD28-mediated costimulation in part by enabling the expression of costimulation-dependent transcription factors. 

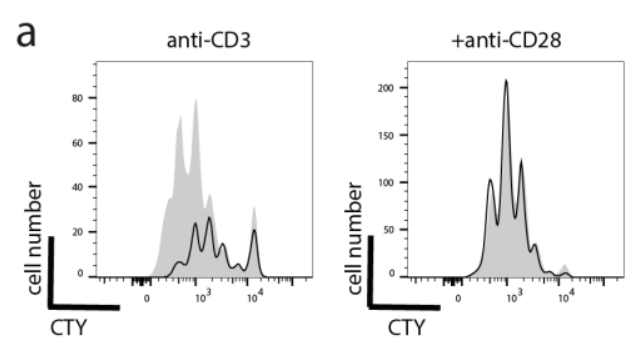

C

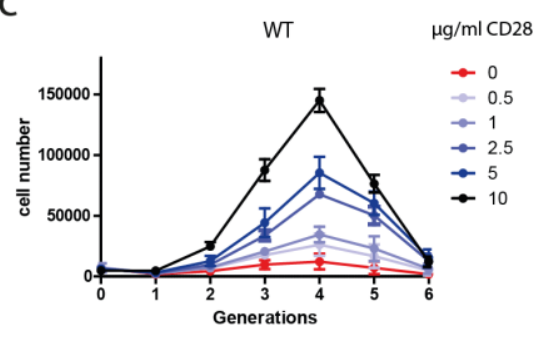

e

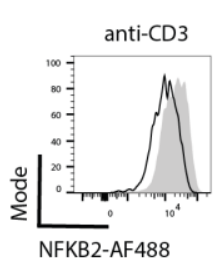

g

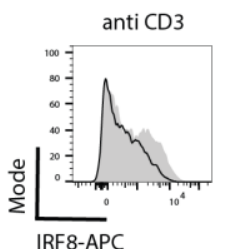

NFKB2

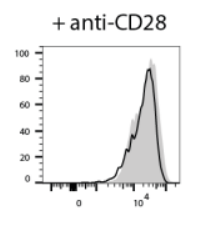

IRF8

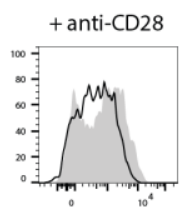

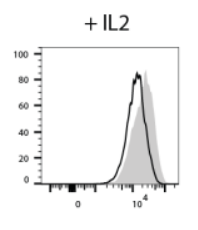

(1)

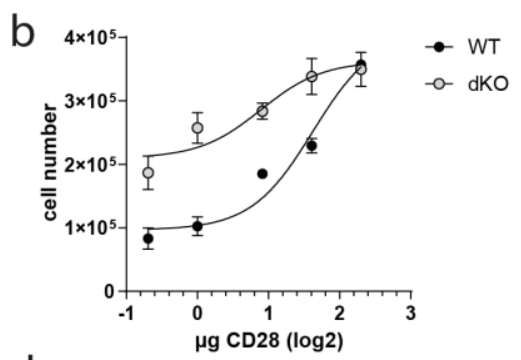

d

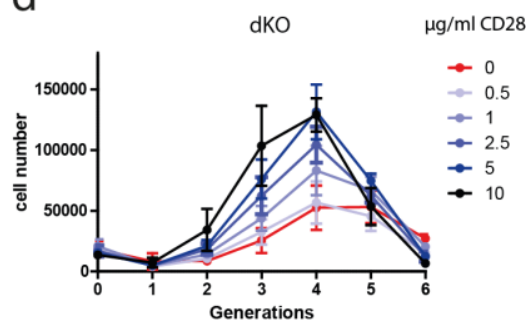

f

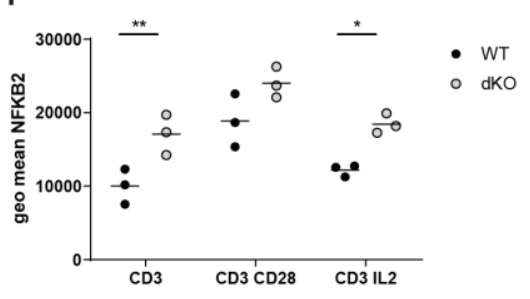

h

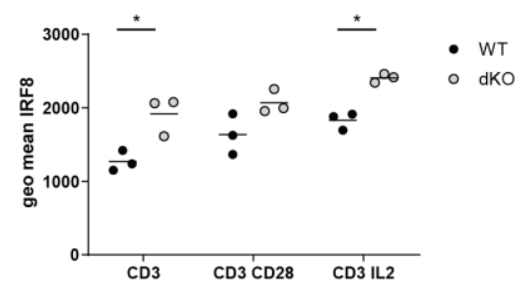

Fig. 7: ZFP36 and ZFP36L1 promote CD28 costimulation dependence for activation. a) CTV dilution by WT (open histograms) and dKO (filled histograms) T cells after 72 hours in the presence of $5 \mu \mathrm{g} / \mathrm{ml}$ plate bound anti CD3 (left panel) and with the addition of $10 \mu \mathrm{g} / \mathrm{ml}$ plate bound anti CD28 (right panel). b) Total numbers of cells recovered with varying amounts of CD28 plate bound added to $5 \mu \mathrm{g} / \mathrm{ml}$ plate bound CD3. Error bars indicate the SEM. c) Cell numbers per generation after 72 hours with plate bound anti CD3 and varying amounts of plate bound anti-CD28 from WT and d) dKO. T cells. Error bars indicate SD. Data is compiled from three biological replicates. e, f) Representative histograms and geo MFI showing expression of NFKB2 and $\mathbf{g}, \mathbf{h}$ ) IRF8 in naive CD8 T cells after 24 hours stimulation with plate bound anti-CD3 in the presence or absence of soluble recombinant IL-2 or plate bound anti-CD28. Open histograms represent WT and filled histograms show dKO cells. Each symbol represents a biological replicate. Statistical significance was determined with two-way ANOVA with Sidak's correction for multiple testing; ${ }^{*}=p<0.05 ;{ }^{* *}=p<0.01$. 


\section{Discussion}

Here we show ZFP36 and ZFP36L1 to act early after CD8 T cell activation to reduce the speed of acquisition of the CTL phenotype and limit the potency of the resulting CTL. Granzyme$B$ and Perforin together with TNF family members have been shown to be the principal mechanisms of CD8 mediated target cell killing ${ }^{38}$. Enhancement of CTL responses underpin why mice in which $\mathrm{T}$ cells lack both RBP are more resilient to infection by IAV and why transfer of naive dKO CD8 T cells into WT mice is sufficient to reduce the body weight loss triggered by IAV. The requirement for the RBP early following the activation of naive CD8 T cells is consistent with the observations of others that the programming of CTL fate happens early after activation ${ }^{4,7-10}$. Our findings reveal an essential role for RBP in limiting this process. The removal of RBP that limit the production of pro-inflammatory cytokines and cytotoxic factors might have been predicted to cause increased immunopathology following IAV infection, however this was not the case possibly because the enhanced CTL response accelerated viral clearance.

Costimulation via CD28 and/or ICOS is critical for the balance of $\mathrm{T}$ cell effector function and tolerance. It is critical not only for successful $\mathrm{T}$ cell activation when antigenic stimulation is suboptimal $^{3}$ but also to install an effector differentiation program which leads to the acquisition of effector functions ${ }^{39}$. The production of IL-2 is one major consequence of costimulation via CD28 and is important for CD8 T cell differentiation and for regulatory $T$ cell function. The NF-KB transcription factors downstream of CD28 act in concert with AP-1 and NFAT to induce the transcription of the $1 / 2$ gene. In addition CD28 dependent signalling promotes the stability of $/ 12 \mathrm{mRNA}$, a finding over 30 years old $^{37}$ for which the mechanisms remains unresolved. Here we show that IL-2 expression is limited by ZFP36 and ZFP36L1 acting at two levels of regulation: Directly via binding to IL-2 mRNA and indirectly by limiting components of the NF-KB pathway ${ }^{35,40}$ that mediate the transcription of the $1 / 2$ gene $^{41}$. This forms the basis for the important regulatory principle of post-transcriptional co-ordination of the production and subsequent fate of $\mathrm{RNA}^{42}$. Our analytical approach focussed on target genes regulated dynamically at the level of RNA six- and 18-hours following activation and will not detect targets that are dynamically regulated earlier than this or for which expression differences are small. Genes in this category include the CLIP targets Nfatc1, Nfatc2 and AP-1 transcription factors Junb and Jund. These have been shown to be critical for $T$ cell activation and IL-2 expression ${ }^{43}$ and may also be part of the regulatory circuit by which ZFP36 and ZFP36L1 limit IL-2. Interestingly, unlike mice with deficiencies in the RBP Roquin, which also regulates costimulation of $T$ cells, we do not observe manifestations of autoimmunity in mice with $T$ cells deficient for ZFP36 and ZFP36L1. This suggests that $\mathrm{T}$ cell effector differentiation and function can be enhanced, without the loss immune regulatory mechanisms which restrain the potentially harmful consequences of an enhanced effector $\mathrm{T}$ cell response.

In addition to Nfkb2 and Rel, ZFP36 and ZFP36L1 regulate Notch1 and Irf8 that are necessary for CD8 effector formation $^{29,33}$. Notch1 has been shown to cooperate with NF-KB transcription factors to induce the expression of EOMES and Granzyme $B^{28,29}$ which we also find increased in the absence of ZFP36 and ZFP36L1 and are key determinants of effector cell function and differentiation. IRF-8 has been shown to promote effector differentiation by integrating antigenic and cytokine signals independently of EOMES andTBET ${ }^{33}$. Its deregulation highlights the systematic nature of repression of factors driving effector differentiation by the ZFP36 family members (Supplementary Figure 6). We did not find evidence of ZPF36 and ZFP36L1 binding or regulating all transcription factors that act early in CD8 T cell differentiation: Batf mRNA is not a target and, while Fos, Tbx21 and Irf4 mRNA were found in CLIP, we found no evidence for increased protein abundance within the first $24 \mathrm{~h}$ (data not shown). However, the ZFP36-family may determine the locations in which mRNAs are translated independently of the amount of protein produced thus impacting on the subcellular distribution of proteins in $\mathrm{CTL}^{44}$. The latter aspect can be of importance in the regulation of the NF-KB pathway and other transcription factors which shuttle between the cytoplasm and nucleus as well as the assembly of multiprotein complexes.

The orderly exit from quiescence requires that growth, cell division and differentiation are coordinated. This starts with the processing of activation signals, the opening of gene-specific chromatin, reprogramming of anabolic metabolism and the induction of transcription factors that trigger differentiation. Considering the different expression-kinetics of target transcripts 
and the dynamic expression profile of ZFP36 and ZFP36L1 we suggest that target mRNAs become "available" for posttranscriptional regulation in a temporally discrete and qualitatively different fashion. Although we validate in the present work only a subset of target transcripts, we propose that ZFP36 and ZFP36L1 exert regulation across multiple cellular processes to limit $\mathrm{T}$ cell activation and differentiation.

\section{Materials and Methods}

\section{Mice}

Mice with single or combined floxed Zfp36 and Zfp36/1 alleles ${ }^{30,45}$, Rosa26 ${ }^{\text {'sl }}$ Cas9-GFP ${ }^{46}$, B6.Cg-Tg(CD4-cre) 1 Cwi mice ${ }^{47}$ and OT-I TCR ${ }^{48}$ (Va2 and V 35 recognizing peptide residues 257-264 of chicken ovalbumin in the context of $\mathrm{H}_{2} \mathrm{~K}^{\mathrm{b}}$ ) were generated on the $\mathrm{C} 57 \mathrm{BL} / 6$ background at the Babraham Institute. The B6.SJL-Ptprc ${ }^{a} P e p c^{b} /$ BoyJ (CD45.1) mice were bred at the Babraham Institute. CD45.1 CD45.2 double positive mice were bred as $\mathrm{F} 1$ from $\mathrm{C} 57 \mathrm{BL} / 6$ (CD45.2) and B6.SJL-Ptprc ${ }^{a}$ Pepc ${ }^{b}$ /BoyJ (CD45.1) mice at the Babraham Institute.

All mouse experimentation was approved by the Babraham Institute Animal Welfare and Ethical Review Body. Animal husbandry and experimentation complied with existing European Union and United Kingdom Home Office legislation and local standards. Mice were bred and maintained in the Babraham Institute Biological Support Unit. Since the opening of this barrier facility (2009), no primary pathogens or additional agents listed in the FELASA recommendations have been confirmed during health monitoring surveys of the stock holding rooms. Ambient temperature was $\sim 19-21^{\circ} \mathrm{C}$ and relative humidity $52 \%$. Lighting was provided on a 12-hour light: 12-hour dark cycle including $15 \mathrm{~min}$ 'dawn' and 'dusk' periods of subdued lighting. After weaning, mice were transferred to individually ventilated cages with 1-5 mice per cage. Mice were fed CRM (P) VP diet (Special Diet Services) ad libitum and received seeds (e.g. sunflower, millet) at the time of cage-cleaning as part of their environmental enrichment.

\section{Infection with Influenza Virus}

8-12-week-old female mice were used for all experiments. During infection animals were anesthetized and infected by intranasal administration with 10-50 PFU of A/Puerto Rico/8/34 (PR8 H1N1) or with $10^{3} \mathrm{PFU}$ of $\mathrm{OVA}_{(257-264)^{-}}$expressing influenza A/WSN/33 (WSN-OVA). Body weights were recorded daily after infection by biological support unit staff. Transfer experiments were performed by staff of the animal support unit in a blinded fashion.

Viral Load determination: RNA was extracted from infected lungs using a Ribopure kit (Ambion, USA) and CDNA was synthesized using MP specific primer: 5'- TCTAACCGAGGTCGAAACGTA -3'. The Real time assay was performed with Platinum Quantitative PCR Super Mix UGD (Invitrogen, USA) by using the following primers and probe specific for Matrix protein (MP) encoding gene of influenza PR8 H1N1 virus strain. Sense primer sequence: 5'-AAGACCAATCCTGTCACCTCTGA -3', antisense primer sequence: 5'- CAAAGCGTCTACGCTGCAGTC -3', and probe sequence: FAM-5'- TTTGTGTTCACGCTCACCGT-3'- TAMRA. Viral loads of the experimental samples were calculated using a standard curve made from a stock PR8 virus with a known concentration of virus plaque forming units per milliliter (PFU/ml).

Adoptive transfer experiments: Splenocytes were incubated for $30 \mathrm{~min}$ at $4^{\circ} \mathrm{C}$ with FITC-conjugated anti-CD4, anti-CD19, anti-NK1.1, anti-F4/80, anti-CD11b, anti-CD11c, anti-MHCII Abs. Next the cells were washed and incubated for $20 \mathrm{~min}$ at $4^{\circ} \mathrm{C}$ with $100 \mu \mathrm{l}$ anti-FITC MACS magnetic beads (Miltenyi Biotec) per $10^{8}$ cells. Cells were washed again and applied to a MACS LS column (Miltenyi Biotec) and the flow through was collected. The resulting cells were $>85 \% \mathrm{CD}^{+} \mathrm{T}$ cells as assessed by flow-

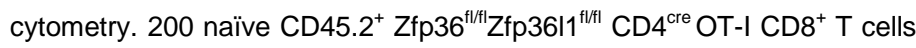

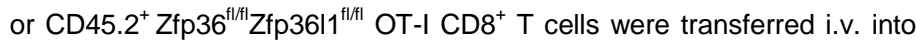
CD $45.1^{+}$recipient mice. One day later the recipient mice were infected with WSN-OVA.

Pulmonary and splenic single cell preparation: Mice were euthanized and lungs were perfused by injecting $5 \mathrm{ml}$ of HBSS $+1 \mathrm{mM}$ EDTA and $25 \mathrm{mM}$ HEPES through the right ventricule of the heart. Single cell suspensions from lungs were prepared with collagenase A (Sigma-Aldrich, USA) $0.5 \mathrm{mg} / \mathrm{ml}$ and DNAse I (Sigma- Aldrich, USA) $0.15 \mathrm{KU} / \mathrm{ml}$ in sterile HBSS with $5 \%$ FBS for 1 hour at $37^{\circ} \mathrm{C}$. The lung digest was filtered and mononuclear cells were re-suspended in 1\% BSA and 1mM EDTA containing PBS (FACS Buffer). Viable cell counts were determined using trypan blue exclusion. Cells were first incubated with Fc block and fixable viability stains (eBioscience) in FACS buffer for 20 min at $4^{\circ} \mathrm{C}$. Cells were washed with FACS buffer and incubated with MHC class I tetramer for 30 $\min$ at $4^{\circ} \mathrm{C}$. After incubation cells were washed with FACS buffer and were further incubated with the fluorochrome conjugated $\mathrm{mAb}$ cocktail. Antiviral CTL responses were quantified by staining with phycoerythrin (PE) labelled $\mathrm{H}-2 \mathrm{D}^{\mathrm{b}}$ MHC class I tetramer loaded with the immunodominant influenza virus nuclear protein (NP) epitope $\mathrm{NP}_{(366-374)}$ (ASNENMETM) (NIH tetramer core facility, USA) or $\mathrm{OVA}_{(257-264)}$ (SIINFEKL) peptide (MBL, USA).

To measure antigen specific cytokine release single cell preparations from lungs were stimulated in the presence of $10 \mu \mathrm{M} \mathrm{NP}{ }_{(366-374)}$ peptide and $1 \mu \mathrm{g} / \mathrm{ml}$ Brefeldin A for 6 hours. Cells were stained for surface markers, fixed and permeabilized with the cytofix/cytoperm reagent (BD Biosciences, USA) and co-stained for intracellular cytokines and GranzymeB. 


\section{Infection with Listeria monocytogenes}

8-12-week old male and female mice were used for all experiments. Bacteria were grown in $\mathrm{BHI}$ medium to an $\mathrm{OD}_{600}$ of 0.1 before each experiment. Mice were infected with a sublethal dose of $5 \times 10^{6} \mathrm{CFU}$ attenuated $(\triangle \mathrm{act} A)$ listeria monocytogenes expressing $\mathrm{OVA}_{(257-264)}{ }^{49}$ by intravenous administration.

Adoptive transfer experiments: naive OT-I cells (if not otherwise stated) were sorted by Flow Cytometry from spleens and $L N$ of mice from a respective genotype and 1000 cells transferred intravenously on the day before infection. In all cotransfer experiments donor cells were mixed with $1 * 10^{6}$ carrier splenocytes of the same genotype as the host.

For characterization of transferred OT-I cells after transfer mice were bled on indicated dates and spleens were collected at final points of analysis. Cells were incubated with $10^{-7} \mathrm{M} \mathrm{N} 4$ peptide for 3 hours in the presence of Brefeldin $A(1 \mu \mathrm{g} / \mathrm{ml})$ in full cell culture medium. After surface staining, cells were fixed with $2 \%$ PFA for 20 min at $4^{\circ} \mathrm{C}$ and permeabilized with BD Perm/wash $+1 \%$ FCS for 20 min at $4^{\circ} \mathrm{C}$, before intracellular Cytokine staining.

\section{Flow Cytometry and monoclonal antibodies}

For cell surface staining single cell suspensions from tissues or cultured cells were prepared in FACS buffer containing PBS 1\% FCS +/- 2mM EDTA (if not otherwise stated in the methods). All cells were blocked with Fcy blocking antibody (24G2, BioXcell) and incubated with fixable cell viability dye eF780 (Thermo Fisher or BD) for $20 \mathrm{~min}$ at $4^{\circ} \mathrm{C}$.

For intracellular staining cells were fixed with BD Cytofix/Cytoperm (554722) or $2-4 \%$ PFA for $20 \mathrm{~min}$ at $4^{\circ} \mathrm{C}$. Cells were permeabilized with BD Permwash (554723) containing $1 \%$ FCS for $20 \mathrm{~min}$ at $4^{\circ} \mathrm{C}$. Intracellular staining was performed in BD Permwash containing $0.5 \%$ FCS and the intracellular antibody cocktail. Cytokine and GranzymeB staining was performed for $1 \mathrm{~h}$ at $4^{\circ} \mathrm{C}$. Intracellular staining for transcription factors was performed for $2 \mathrm{~h}$ at RT. Staining for intracellular Notch1-IC was performed using fixation and permeabilization with Foxp3 transcription factor staining kit (Invitrogen; 00-5523-00). Intracellular staining was performed at room temperature in BD Foxp3 permeabilization buffer.

The following antibody clones were used in the flow cytometry experiments: CD4 (GK1.5,RM4-5), CD8 (53-6.7), CD45.1 (A20), CD45.2 (104), CD45 (30-F11), KLRG1 (2F1), CD127 (A7R34), CD44 (IM7), CD25 (7D4, PC61), CD69 (H1.2F3), MHCII ( M5/11415.2), TCRß (H57597), CD11c (cN418), F4/80 (BM8), NK1.1 (PK136), CD19 (6D5), Thy1.1 (OX7), TNF (MP6-XT22), IFN-y (XMG1.2), IL2 (JES6-5H4), GranzymeB (GB11), c-REL (1RELAH5), NFKB2 (EPR18756), IRF8 (V3GYWCH), NOTCH1-IC (mN1A), EOMES (Dan11mag). Secondary antibodies: polyclonal goat anti Rabbit (H+L) AF488 (A11034, Invitrogen). Data was acquired using a Fortessa Flow Cytometer equipped with $355 \mathrm{~nm}, 405 \mathrm{~nm}$, 488nm, 561nm and 640nm lasers (Beckton Dickinson). Flow Cytometry data was analysed using FlowJo 10.6 software.

\section{In vitro culture and activation of T cells}

All in vitro and cell culture experiments were performed in IMDM culture medium (Gibco) supplemented with 10\% FCS, GlutaMAX ${ }^{\mathrm{TM}}$ (Gibco),

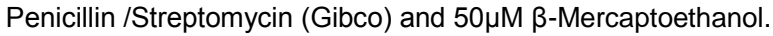

Naive CD8 T cells were isolated from Spleen and Lymph Nodes using two rounds of negative depletion with Streptavidin DynaBeads ${ }^{\mathrm{TM}}$ (Thermo Fisher) using $1^{*} 10^{8}$ and $4^{*} 10^{7}$ beads per $1 * 10^{8}$ total splenocytes/LN cells. The following antibodies were used for the depletion cocktail: B220-Bio (RA3-6B3), CD4-Bio (GK1.5), CD11b-Bio (M1/70), CD11c-Bio (N418), CD19-Bio (1D3), CD44-Bio (IM7), CD105-Bio, F4/80-Bio (BM8), GR1-Bio (RB6-8C5), NK1.1-Bio (PK136), Ter119-Bio (Ter-119), yסTCR-Bio (GL3). Cells were stimulated at a starting concentration of $5^{\star} 10^{5}$ cells $/ \mathrm{ml}$, if not otherwise stated with $5 \mu \mathrm{g} / \mathrm{ml}$ plate bound anti-CD3 (145-2C11, BioXcell) and $1 \mu / \mathrm{ml}$ plate bound anti-CD28 (37.51, BioXcell). OT-I transgenic naive CD8 $\mathrm{T}$ cells were stimulated with (N4) SIINFEKL peptide at $10^{-10} \mathrm{M}$. In some conditions recombinant mouse IL-2 (Peprotech) was added at $20 \mathrm{nM}$. For the analysis of proliferation, cells were labelled with cell trace violet (Thermo Fisher) or cell trace yellow (Thermo Fisher) at $10 \mu \mathrm{M}$ final concentration for $6 \mathrm{~min}$ at $37^{\circ} \mathrm{C}$, before stimulation. Cell numbers per generation were enumerated using cell counting beads for flow cytometry (ACBP-50-10, Spherotech).

For the in vitro generation of CTLs $1^{*} 10^{6}$ cells per $\mathrm{ml}$ of total splenocytes/LN cells from OT-I mice were activated with $10^{-7} \mathrm{M}$ (N4) SIINFEKL peptide in the presence of $20 \mathrm{nM} \mathrm{IL-2}$ and $2 \mathrm{nM} \mathrm{IL-12} \mathrm{for} \mathrm{two}$ days. After 1-2 days of stimulation, cells were maintained in 20nM IL-2 for seven days.

\section{Cytokine assays}

Mouse IL-2 secretion assay was performed following manufacturer's instructions (130-090-491; Miltenyi Biotech). In brief; $5^{\star} 10^{5} / \mathrm{ml}$ purified naive OT-I cells were activated with $10^{-10} \mathrm{~N} 4$ peptide in cell culture medium for the indicated timepoints. Cells were washed twice and incubated with capture reagent for $1 \mathrm{~h}$ at $37^{\circ} \mathrm{C}$ while mixing the cells every $10 \mathrm{~min}$. Cells were surface stained with antibody cocktail containing anti IL2-PE antibody.

\section{Cytotoxicity assays}

In vivo: Splenic leukocytes from naïve C57BL/6 mice were pulsed for $1 \mathrm{hr}$ with $10 \mu \mathrm{g} / \mathrm{ml}$ influenza virus $\mathrm{NP}_{(366-374)}$ peptide and were subsequently labeled for $10 \mathrm{~min}$ at $37^{\circ} \mathrm{C}$ with $5 \mu \mathrm{M}$ CFSE (Invitrogen) (CFSE ${ }^{\text {high }}$; loaded cells). Non-peptide loaded control cells were labelled with $0.5 \mu \mathrm{M}$ CFSE $^{\text {low }}$. The two populations of cells were then mixed together in a 1:1 ratio and $6 \times 10^{6}$ cells were transferred intravenously into mice that had previously been infected with influenza PR8 ten days before, or 
transferred into uninfected control mice. Mice were killed 1 hour later and the percentage of peptide loaded target cells and peptide non-loaded cells was quantified by flow cytometry.

In vitro: OT-I CTLs were cultured in the presence of N4 peptide -pulsed (target), and non-pulsed (non-target) EL4 mouse cell line. Target cells were pulsed with $10^{-7} \mathrm{M} \mathrm{N} 4$ peptide for $30 \mathrm{~min}$ at $37^{\circ} \mathrm{C}$ and washed $1 \mathrm{x}$ with ice cold FBS and $2 x$ with cell culture medium. Target and non-target cells were marked with CTY and CTV respectively at $10 \mu \mathrm{M}$ final concentration. Killing experiments were performed at different ratios of target versus OT-I cells for 3 hours at $37^{\circ} \mathrm{C}$.

The percentage of target cell killing was determined as: 100\{(percentage of peptide-pulsed targets in infected recipients/percentage of un-pulsed targets in infected recipients)/ (percentage of peptide-pulsed targets in naïve recipients/un-pulsed targets in naïve recipients) $X 100\}$.

\section{CrispR Cas9 experiments and sgRNA cloning}

MSCV_hU6_Bbsl-ccdB-Bbsl_iScaffold_mPGK_puro-2A-Thy1.1

(MSCV_sgRNA_puro-Thy1.1) was generated from vectors MIGRI and PKLV2_sgRNA_puro-Thy1.1. For individual sgRNAs two 24nt oligonucleotides were annealed and ligated (T4) into a Bbsl linearized MSCV_sgRNA_puro-Thy1.1 vector. Pairwise sgRNAs were initially independently cloned alongside either the hU6 or mU6 promoter then amplified by PCR and ligated in tandem into the destination vector. Plat-E cells at $0.3^{*} 10^{6}$ cells/well of 12 -well plate were seeded and cultured overnight before transfection with $100 \mu$ l OptiMEM, $3 \mu$ l TransIT®-293, $0.8 \mu \mathrm{g}$ transfer vector, $0.2 \mu \mathrm{g}$ packaging $\mathrm{pCL}-\mathrm{ECO}$. Retrovirus was harvested and frozen from transfected plat-E cells over the subsequent 72 hours.

For CRISPR/Cas9-gene editing experiments $1 * 10^{6}$ cells per mltotal splenocytes/LN cells from OT-I mice expressing Cas9 endonuclease (OT$\mathrm{I}^{+}$; CD4 ${ }^{\text {cre }}$; Rosa26Isl; Cas9-GFP) were activated with $10^{-7} \mathrm{M}$ (N4) SIINFEKL peptide in the presence of $20 \mathrm{nM} \mathrm{IL-2}$ and $2 \mathrm{nM} \mathrm{IL-12}$ for $24 \mathrm{~h}$. Plasmids encoding single guide RNAs (sgRNAs) were introduced by retroviral transduction into activated OT-I cells by centrifugation at $(1000 \mathrm{xg})$ for 45 minutes at $32^{\circ} \mathrm{C}$. Following transduction OT-I cells were maintained with exogenous IL2 for $24 \mathrm{~h}$. Transduced cells were isolated by sorting cells expressing Thy1.1 (CD90.1) transduction marker. Cells were maintained for a further 4 days with exogenous IL-2 to generate CTLs.

To test for the efficiency of CRISPR/Cas9-induced knock out of Zfp36 and Zfp36/1 CTLs were stimulated for $3 \mathrm{~h}$ with $10^{-7} \mathrm{M} \mathrm{N} 4$, and expression of Zfp36 family members was detected by Western blotting. Guide RNA combinations to target individual ZFP36 family members were used as follows:
Non-targeting guides:

GGTAGAGTCGCGAACGCTAC; GATGCGGACCCACGTTAAGC; GGCATGGAGTCAACGTCCGC

Guides targeting both Zfp36 and Zfp36/1

GAGCGGGCGTTGTCGCTACG + GGAGTGACCGAGTGCCTGCG; GAGCGGGCGTTGTCGCTACG + GTTGAGCATCTTGTTACCCT; GATGACCTGTCATCCGACCA + GTTGAGCATCTTGTTACCCT;

\section{Western Blotting}

Whole cell lysates were prepared using 1x RIPA buffer [SDS $0.1 \%(\mathrm{w} / \mathrm{v})$, Sodium deoxycholate $0.4 \%$ (w/v), NP-40 0.5\% (v/v), 100mM NaCl, 50mM Tris-HCl, pH 7.4], supplemented with 1:100 protease inhibitor cocktail (Sigma P8340), phosphatase inhibitor cocktail III (Sigma P004), and 2 $\mathrm{U} / \mathrm{ml}$ Benzonase (9025-65-4). Protein lysate concentration was determined using Pierce BCA protein assay kit (23225). Lysates were denatured for $5 \mathrm{~min}$ at $97^{\circ} \mathrm{C}$ with Laemmli buffer containing $5 \% \quad \beta 2$ mercaptoethanol, and the equivalent of $30 \mu \mathrm{g}$ of total protein was loaded per lane. Samples were resolved by $10 \%$ SDS-PAGE and transferred to nitrocellulose membrane using iBlot2 transfer device (IB21001). The membrane was probed with anti-ZFP36 mouse monoclonal (Origene \#OTI3D10) $(2 \mu \mathrm{g} / \mathrm{ml}$ ) and anti-ZFP36L1 polyclonal (CST \#BRF1/2) (32 $\mathrm{ng} / \mathrm{ml}$ ). Proteins were detected by incubating membrane with anti-Mouse IgG IRDye800CM (Licor \#926-32210) and anti-Rabbit IgG IRDye680RD (Licor \#925-68071), and scanning the membrane with Licor Odyssey CLx using standard methods. GAPDH was detected using rabbit monoclonal anti-GAPDH (CTS \#5174) at 1:1,000 and anti-Rabbit IgG IRDye680RD. Image analysis was conducted using ImageStudio Lite version 5.2, and normalized protein signal was calculated using standard methods.

\section{ZFP36L1 iCLIP analysis}

$25^{\star} 10^{6}$ in vitro- generated CTLs from three biological replicates were crosslinked at $150 \mathrm{~mJ} / \mathrm{cm}^{2}$ with Stratalinker 2400 with $245 \mathrm{~nm}$ radiation, and snap frozen on dry ice. Cells were then lysed and sonicated, and treated with RNase I $(0.03 \mathrm{U} / \mathrm{ml})$ [AM2294] and Turbo DNase (2 U/ml) [AM2238] at $37^{\circ} \mathrm{C}$ for $3 \mathrm{~min}$ at $1100 \mathrm{rpm}$. Anti-ZFP36L1 polyclonal antibody $(2 \mu \mathrm{g} / \mathrm{ml})$ [ABN-192] was pre-coupled to protein $A / G$ Dynabeads [Invitrogen 88802], and incubated with lysate under rotation overnight at $4^{\circ} \mathrm{C}$. ZFP36L1-RNA complexes were magnetically separated, and RNA was de-phosphorylated using FastAP Alkaline phosphatase [Invitrogen EF0651] for $40 \mathrm{~min}$ at $37^{\circ} \mathrm{C}$ at $1100 \mathrm{rpm}$. IRDye-linked pre-adenylated adaptors were ligated to RNA using T4 RNA ligase I (M0437M), in the presence of $11.25 \%$ PEG8000, at room temperature for 75 min. Nonligated adaptors were removed using RecJF 5'-3' exonuclease (M0264S) for $1 \mathrm{~h}$ at $30^{\circ} \mathrm{C}$ at $1100 \mathrm{rpm}$, and RNA was de-adenylaed using NEB deadenylase (M0331S) for $30 \mathrm{~min}$ at $37^{\circ} \mathrm{C}$ at $1100 \mathrm{rpm}$. ZFP36L1-RNA 
complexes were removed from magnetic beads by re-suspending in NuPAGE LDS buffer (sup with $100 \mathrm{mM}$ DTT), and heating to $70^{\circ} \mathrm{C}$ for 1 min and shaking at $1100 \mathrm{rpm}$. The supernatant was loaded onto pre-cast 4-12\% NuPAGE Bis-Tris gel [Invitrogen] and resolved by running for 65 min at $180 \mathrm{~V}$ with MOPS buffer (NP0001). Complexes were transferred to nitrocellulose membrane using XCell II Blot module [Invitrogen] for $1 \mathrm{~h}$ at $30 \mathrm{~V}$ using standard methodology. Nitrocellulose membrane was scanned using Licor Odyssey CLx and ZFP36L1-RNA complexes excised from membrane by cutting $35-55 \mathrm{kDa}$ above expected running weight of ZFP36L1. Protein was digested by incubating membrane at $50^{\circ} \mathrm{C}$ for $1 \mathrm{~h}$ with proteinase $\mathrm{K}$ (Merck) diluted in PK-SDS buffer. Supernatant was mixed with phenol:chloroform:isoamyl alcohol and transferred to $2 \mathrm{ml}$ phase-lock gel tube and spun for $5 \mathrm{~min}$ at $17,000 \mathrm{xg}$ at room temperature. Chloroform was added to the top phase and samples were centrifuged for $5 \mathrm{~min}$ at $17,000 \mathrm{xg}$ at $4^{\circ} \mathrm{C}$. Aqueous phase was isolated and RNA precipitated over night at $-20^{\circ} \mathrm{C}$ with $100 \%$ Ethanol, $1 \mathrm{M} \mathrm{NaCl}$ and Glycoblue $(0.1 \% \mathrm{v} / \mathrm{v})$ [AM9515]. Reverse transcription was conducted using Superscript IV [Invitrogen 18090010] and $1 \mathrm{pmol} / \mu \mathrm{I}$ irCLIP_ddRT primers featuring $5 \mathrm{nt}$ unique molecular identifiers. cDNA was purified using AMPure XP beads [Beckman Coulter A63881] and circularized in the presence of Beatine (1 M) using CircLigase II [Lucigen CL9021K]. PCR amplification was conducted using P5 and P3 solexa primers [lllumina] and Phusion HF master mix [NEB M0536S]. PCR products were run on $6 \%$ TBE gel and DNA in the range of $145-400 \mathrm{nt}$ isolated using standard methodology. iCLIP libraries were multiplexed and sequenced using HiSeq2500-RapidRun (100bp Single End).

\section{ZFP36L1 iCLIP mapping}

iCLIP data was mapped to GRCm38 mouse genome assembly using bowtie2. Barcoded adaptors of iCLIP sequenced reads were removed before mapping, crosslink sites were defined by the nucleotide preceding iCLIP cDNAs using Genialis iMaps web server: https://imaps.genialis.com/iclip.

\section{Data and statistical analysis}

HITS-CLIP datasets for ZFP36 in CD4 T cells following $4 \mathrm{~h}$ or $72 \mathrm{~h}$ activation were obtained from GSE96074 ${ }^{20}$. This data, together with the iCLIP data, were processed using the Genialis iMaps web server (https://imaps.genialis.com/iclip). Data was deduplicated based on the random barcodes, trimmed with Cutadapt ${ }^{50}$, and mapped to GRCm38 mouse genome assembly using STAR ${ }^{51}$. Significant crosslink sites, defined by the nucleotide preceding iCLIP or HITS-CLIP cDNAs, were identified using the iCount pipeline (https://icount.readthedocs.io/en/latest/index.html).

For CLIP target gene identification, target transcripts were filtered for the presence of identical significant crosslink sites in their 3'UTRs in at least 3 out of 4 or 5 (for ZFP36) and 2 out of 3 (for ZFP36L1) biological replicates. Unique and common targets identified from the two datasets were then unified and taken as our final list of high confidence ZFP36/L1 target genes.

RNA-seq data for naïve and in-vitro activated CD8 T cells were obtained from GSE $77857^{26}$. Data were trimmed using Trim Galore (https://www.bioinformatics.babraham.ac.uk/projects/trim_galore), mapped to the GRCm38 mouse genome build using Hisat $2^{52}$, taking into account known splice sites from the Ensembl Mus_musculus.GRCm38.90 annotation release. Raw read counts over mRNA features from the same annotation release were quantified using Seqmonk (https://www.bioinformatics.babraham.ac.uk/projects/seqmonk). Differentially expressed genes at $6 \mathrm{~h}$ and $18 \mathrm{~h}$ after CD8 $\mathrm{T}$ cells activation were identified using DESeq2 analysis with default parameters for each time point relative to $0 \mathrm{~h}$, and were selected for adj. pvalue $<=0.05$ and $1.3<\log 2 \mathrm{FC}<-1.3$ (using 'normal' log2 fold change shrinkage). Genes with a lower baseMean expression than 30 normalized read counts were excluded from analysis.

For analysis of binding site conservation, a 70nt sequence around the identified CLIP binding site was analyzed for conservation among vertebrates using the UCSC genome browser.

Statistical analysis was performed with Graph Pad Prism 8.1.2.

\section{Author Contributions and Notes}

G.P. conceptualization; methodology; investigation; validation; formal analysis; visualisation; writing original draft preparation; T.J. M. conceptualization; methodology; investigation; validation; formal analysis; visualisation; writing review and editing; K.C. conceptualization methodology; investigation; visualisation; writing review and editing; S.E.B. conceptualization; resources; methodology; writing review and editing; V. D'A. investigation; writing review and editing; L.M. software; formal analysis; investigation; visualisation; writing review and editing; D.J.T. methodology; investigation; writing review and editing; O.G. investigation; formal analysis and editing; F. S. methodology; writing review and editing; P.D.K. conceptualization; resources; writing review and editing; M.T. Conceptualisation, supervision, funding acquisition, writing review and editing.

The authors declare no conflict of interest.

\section{Acknowledgments}

We thank the Babraham Institute Biological Support Unit, Flow Cytometry and Kirsty Bates, Anne Segonds-Pichon and the Bioinformatics Facilities 
bioRxiv preprint doi: https://doi.org/10.1101/2021.06.03.446738; this version posted June 4, 2021. The copyright holder for this preprint (which was not certified by peer review) is the author/funder, who has granted bioRxiv a license to display the preprint in perpetuity. It is made available under aCC-BY 4.0 International license.

for assistance; Elisa Monzon-Casanova, Adrian Liston, Sarah Ross and Arianne Richard for comments on the manuscript. This study was supported by funding from the Biotechnology and Biological Sciences Research Council (BBSRC) (BBS/E/B/000C0407; BBS/E/B/000C0428; the BBSRC Core Capability Grant to the Babraham Institute; and a Wellcome Investigator award (200823/Z/16/Z) to M.T. FS was supported by European Molecular Biology Organization (EMBO) Long-Term Fellowship (ALTF 880-2018). T.J.M. was supported by the BBSRC Cambridge doctoral training partnership. V. D'A. was supported by the Cambridge Trust.

\section{References}

1. Bennett, T. J., Udupa, V. A. V. \& Turner, S. J. Running to Stand Still: Naive CD8+ T Cells Actively Maintain a Program of Quiescence. IJMS 21, 9773 (2020).

2. Gett, A. V., Sallusto, F., Lanzavecchia, A. \& Geginat, J. T cell fitness determined by signal strength. Nat Immunol 4, 355-360 (2003).

3 . Hünig, T., Beyersdorf, N. \& Kerkau, T. CD28 co-stimulation in T-cell homeostasis: a recent perspective. ITT 111 (2015) doi:10.2147/ITT.S61647.

4. Obst, R. The Timing of $\mathrm{T}$ Cell Priming and Cycling. Front. Immunol. 6, (2015)

5. Zehn, D., Lee, S. Y. \& Bevan, M. J. Complete but curtailed Tcell response to very low-affinity antigen. Nature 458, 211-214 (2009).

6. Richard, A. C. et al. T cell cytolytic capacity is independent of initial stimulation strength. Nat Immunol 19, 849-858 (2018).

7. Chiu, C. et al. Early acquisition of cytolytic function and transcriptional changes in a primary CD8+ T-cell response in vivo. Blood 109, 1086-1094 (2007).

8. Prlic, M., Hernandez-Hoyos, G. \& Bevan, M. J. Duration of the initial TCR stimulus controls the magnitude but not functionality of the CD8+ T cell response. Journal of Experimental Medicine 203, 2135-2143 (2006).

9. van Stipdonk, M. J. B. et al. Dynamic programming of CD8+ T lymphocyte responses. Nat Immunol 4, 361-365 (2003).

10. van Stipdonk, M. J. B., Lemmens, E. E. \& Schoenberger, S. P. Naïve CTLs require a single brief period of antigenic stimulation for clonal expansion and differentiation. Nat Immunol 2, 423-429 (2001).

11. Pipkin, M. E. et al. Interleukin-2 and Inflammation Induce Distinct Transcriptional Programs that Promote the Differentiation of Effector Cytolytic T Cells. Immunity 32, 79-90 (2010).

12. Starbeck-Miller, G. R., Xue, H.-H. \& Harty, J. T. IL-12 and type I interferon prolong the division of activated CD8 T cells by maintaining high-affinity IL-2 signaling in vivo. The Journal of Experimental Medicine 211, 105-120 (2014).

13. Joshi, N. S. et al. Inflammation Directs Memory Precursor and Short-Lived Effector CD8+ T Cell Fates via the Graded Expression of Tbet Transcription Factor. Immunity 27, 281-295 (2007).

14. Kaech, S. M. \& Cui, W. Transcriptional control of effector and memory CD8+ T cell differentiation. Nat Rev Immunol 12, 749-761 (2012).

15. Henning, A. N., Roychoudhuri, R. \& Restifo, N. P. Epigenetic control of CD8+ T cell differentiation. Nat Rev Immunol 18, 340-356 (2018).

16. Pipkin, M. E. Runx proteins and transcriptional mechanisms that govern memory CD8 T cell development. Immunol. Rev. 300, 100124 (2021).

17. Salerno, F., Turner, M. \& Wolkers, M. C. Dynamic PostTranscriptional Events Governing CD8+ T Cell Homeostasis and Effector Function. Trends in Immunology 41, 240-254 (2020).

18. Chang, P.-P. et al. Breakdown in Repression of IFN-y mRNA Leads to Accumulation of Self-Reactive Effector CD8 ${ }^{+}$T Cells. J.I. 189, 701-710 (2012).
19. Fu, M. \& Blackshear, P. J. RNA-binding proteins in immune regulation: a focus on $\mathrm{CCCH}$ zinc finger proteins. Nat Rev Immunol 17, 130-143 (2017).

20. Moore, M. J. et al. ZFP36 RNA-binding proteins restrain T cell activation and anti-viral immunity. eLife 7, e33057 (2018).

21. Bell, S. E. et al. The RNA binding protein Zfp36/1 is required for normal vascularisation and post-transcriptionally regulates VEGF expression. Dev. Dyn. 235, 3144-3155 (2006).

22. Ma, W. \& Mayr, C. A Membraneless Organelle Associated with the Endoplasmic Reticulum Enables 3'UTR-Mediated Protein-Protein Interactions. Cell 175, 1492-1506.e19 (2018)

23. Sneezum, L. et al. Context-Dependent IL-1 mRNADestabilization by TTP Prevents Dysregulation of Immune Homeostasis Under Steady State Conditions. Front. Immunol. 11, 1398 (2020).

24. Wang, Q. et al. Tristetraprolin inhibits macrophage IL-27induced activation of antitumour cytotoxic T cell responses. Nat Commun 8, 867 (2017)

25. Salerno, F. et al. Translational repression of pre-formed cytokine-encoding mRNA prevents chronic activation of memory T cells. Nat Immunol 19, 828-837 (2018).

26. Roychoudhuri, $\mathrm{R}$. et al. $\mathrm{BACH} 2$ regulates $\mathrm{CD} 8+\mathrm{T}$ cell differentiation by controlling access of AP-1 factors to enhancers. Nat Immunol 17, 851-860 (2016).

27. Assenmacher, M., Scheffold, A. \& Radbruch, A. Cytometric cytokine secretion assay: Detection and isolation of cytokine-secreting $T$ cells. in Methods in Microbiology vol. 32 59-75 (Elsevier, 2002).

28. Knudson, K. M. et al. NFKB-Pim-1-Eomesodermin axis is critical for maintaining CD8 T-cell memory quality. Proc Natl Acad Sci USA 114, E1659-E1667 (2017).

29. Cho, O. H. et al. Notch Regulates Cytolytic Effector Function in CD8 ${ }^{+}$T Cells. J Immunol 182, 3380-3389 (2009).

30. Hodson, D. J. et al. Deletion of the RNA-binding proteins ZFP36L1 and ZFP36L2 leads to perturbed thymic development and T lymphoblastic leukemia. Nat Immunol 11, 717-724 (2010).

31. Cruz-Guilloty, F. et al. Runx3 and T-box proteins cooperate to establish the transcriptional program of effector CTLs. Journal of Experimental Medicine 206, 51-59 (2009).

32. Pearce, E. L. Control of Effector CD8+ T Cell Function by the Transcription Factor Eomesodermin. Science 302, 1041-1043 (2003).

33. Miyagawa, F. et al. Interferon regulatory factor 8 integrates Tcell receptor and cytokine-signaling pathways and drives effector differentiation of CD8 T cells. Proc Natl Acad Sci USA 109, 12123-12128 (2012).

34. Nayar, R. et al. Graded Levels of IRF4 Regulate CD8 ${ }^{+}$T Cell Differentiation and Expansion, but Not Attrition, in Response to Acute Virus Infection. J.I. 192, 5881-5893 (2014).

35. Giardino Torchia, M. L., Conze, D. B., Jankovic, D. \& Ashwell, J. D. Balance between NF-KB p100 and p52 Regulates T Cell Costimulation Dependence. J.I. 190, 549-555 (2013).

36. Kane, L. P., Lin, J. \& Weiss, A. It's all Rel-ative: NF-KB and CD28 costimulation of T-cell activation. Trends in Immunology 23, 413420 (2002)

37. Lindstein, T., June, C., Ledbetter, J., Stella, G. \& Thompson, C. Regulation of lymphokine messenger RNA stability by a surfacemediated T cell activation pathway. Science 244, 339-343 (1989).

38. Hufford, M. M., Kim, T. S., Sun, J. \& Braciale, T. J. The effector $\mathrm{T}$ cell response to influenza infection. Curr Top Microbiol Immunol 386, 423-455 (2015).

39. Nurieva, R. et al. T-cell tolerance or function is determined by combinatorial costimulatory signals. EMBO J 25, 2623-2633 (2006).

40. Matsumoto, M. et al. Essential Role of NF-KB-Inducing Kinase in T Cell Activation Through the TCR/CD3 Pathway. $J$ Immunol 169 1151-1158 (2002).

41. Rao, S., Gerondakis, S., Woltring, D. \& Shannon, M. F. c-Rel Is Required for Chromatin Remodeling Across the IL-2 Gene Promoter. $J$ Immunol 170, 3724-3731 (2003).

42. Turner, M., Galloway, A. \& Vigorito, E. Noncoding RNA and its associated proteins as regulatory elements of the immune system. Nature Immunology 15, 484-491 (2014). 
bioRxiv preprint doi: https://doi.org/10.1101/2021.06.03.446738; this version posted June 4, 2021. The copyright holder for this preprint (which was not certified by peer review) is the author/funder, who has granted bioRxiv a license to display the preprint in perpetuity. It is made available under aCC-BY 4.0 International license.

Petkau et al., 04062021

43. Papavassiliou, A. G. \& Musti, A. M. The Multifaceted Output of c-Jun Biological Activity: Focus at the Junction of CD8 T Cell Activation and Exhaustion. Cells 9, 2470 (2020).

44. Mayr, C. What Are 3' UTRs Doing? Cold Spring Harb Perspect Biol 11, (2019).

45. Newman, R. et al. Maintenance of the marginal-zone B cell compartment specifically requires the RNA-binding protein ZFP36L1. Nat Immunol 18, 683-693 (2017).

46. Platt, R. J. et al. CRISPR-Cas9 Knockin Mice for Genome Editing and Cancer Modeling. Cell 159, 440-455 (2014).

47. Lee, P. P. et al. A Critical Role for Dnmt1 and DNA Methylation in T Cell Development, Function, and Survival. Immunity 15, 763-774 (2001).

48. Hogquist, K. A. et al. T cell receptor antagonist peptides induce positive selection. Cell 76, 17-27 (1994).

49. Pearce, E. L. \& Shen, H. Generation of CD8 T Cell Memory Is Regulated by IL-12. J Immunol 179, 2074-2081 (2007).

50. Martin, M. Cutadapt removes adapter sequences from highthroughput sequencing reads. EMBnet j. 17, 10 (2011).

51. Dobin, A. et al. STAR: ultrafast universal RNA-seq aligner. Bioinformatics 29, 15-21 (2013).

52. Kim, D., Langmead, B. \& Salzberg, S. L. HISAT: a fast spliced aligner with low memory requirements. Nat Methods 12, 357-360 (2015). 